\title{
Crescerin uses a TOG domain array to regulate microtubules in the primary cilium
}

\author{
Alakananda Das, ${ }^{a, b}$, Daniel J. Dickinson ${ }^{c, d}$, Cameron C. Wood c, Bob Goldstein ${ }^{c, d}$, and Kevin C. Slep \\ aDepartment of Biochemistry and Biophysics, bMolecular and Cellular Biophysics Program, 'Department of Biology, \\ and 'Lineberger Comprehensive Cancer Center, University of North Carolina, Chapel Hill, NC 27599
}

\begin{abstract}
Eukaryotic cilia are cell-surface projections critical for sensing the extracellular environment. Defects in cilia structure and function result in a broad range of developmental and sensory disorders. However, mechanisms that regulate the microtubule (MT)-based scaffold forming the cilia core are poorly understood. TOG domain array-containing proteins ch-TOG and CLASP are key regulators of cytoplasmic MTs. Whether TOG array proteins also regulate ciliary MTs is unknown. Here we identify the conserved Crescerin protein family as a cilia-specific, TOG array-containing MT regulator. We present the crystal structure of mammalian Crescerin1 TOG2, revealing a canonical TOG fold with conserved tubulin-binding determinants. Crescerin1's TOG domains possess inherent MT-binding activity and promote MT polymerization in vitro. Using Cas9-triggered homologous recombination in Caenorhabditis elegans, we demonstrate that the worm Crescerin family member $\mathrm{CHE}-12$ requires TOG domain-dependent tubulin-binding activity for sensory cilia development. Thus, Crescerin expands the TOG domain array-based MT regulatory paradigm beyond ch-TOG and CLASP, representing a distinct regulator of cilia structure.
\end{abstract}

\author{
Monitoring Editor \\ Samara Reck-Peterson \\ Harvard Medical School \\ Received: Aug 28, 2015 \\ Accepted: Sep 11, 2015
}

\section{INTRODUCTION}

Eukaryotic cilia and flagella are specialized microtubule (MT)-based organelles. Motile cilia are classically associated with cellular movement or direction of extracellular fluid flow (Satir and Christensen, 2008). In addition, most mammalian cell types contain a primary cilium - a solitary, antenna-like extension that receives extracellular chemical and mechanical stimuli and transduces a signal to the cell body to elicit a response (Marshall and Nonaka, 2006; Satir and Christensen, 2007; Christensen et al., 2008; Veland et al., 2009; Hoey et al., 2012). Genetic mutations in ciliary proteins underlie diseases collectively termed ciliopathies. In many of these diseases,

This article was published online ahead of print in MBoC in Press (http://www .molbiolcell.org/cgi/doi/10.1091/mbc.E15-08-0603) on September 16, 2015. Address correspondence to: Kevin C. Slep (kslep@bio.unc.edu).

A.D., D.J.D., B.G., and K.C.S. conceived the research; A.D., D.J.D., and C.W., collected data; A.D. and D.J.D. analyzed data; and A.D. and K.C.S. wrote the manuscript. All authors commented on the manuscript.

Abbreviations used: CD, circular dichroism; HR, HEAT repeat; MAP, microtubuleassociated protein; MT, microtubule; WT, wild type.

(C) 2015 Das et al. This article is distributed by The American Society for Cell Biology under license from the author(s). Two months after publication it is available to the public under an Attribution-Noncommercial-Share Alike 3.0 Unported Creative Commons License (http://creativecommons.org/licenses/by-nc-sa/3.0). "ASCB ${ }^{\circledR}$," "The American Society for Cell Biology ${ }^{\circledR}$," and "Molecular Biology of the Cell ${ }^{\circledR} "$ are registered trademarks of The American Society for Cell Biology. the structural integrity of the primary cilium is compromised (Marshall, 2008).

The structural core of the cilium and flagellum is the axoneme, composed of parallel MTs arranged in ninefold radial symmetry. The axonemal MTs are polymers of $\alpha \beta$-tubulin heterodimers. Nine axonemal doublet MTs arise from the basal body, a modified centriole docked at the plasma membrane, which templates the radial MT symmetry and anchors the MT minus ends. The MT doublets, composed of $A$ and $B$ tubules, span the length of the cilia middle zone. At the middle/distal zone transition, $B$ tubules terminate, and singlet A tubules extend into the distal zone. The axonemal MTs provide structural support, serve as tracks along which anterograde and retrograde intraflagellar transport complexes traffic ciliary components, and, in motile cilia, serve as the substrate for axonemal dynein motors that drive motility (Scholey, 2003, 2008; Ishikawa, 2012; Kobayashi and Takeda, 2012). Doublet MTs in the middle zone are stable and resistant to MT-depolymerizing agents, including colchicine (Tilney and Gibbins, 1968). In contrast, singlet MTs in the distal segment are dynamic and change their length in response to external stimuli (Marshall and Rosenbaum, 2001; Mukhopadhyay et al., 2008; Rich and Clark, 2012). The plus ends of both the A and B tubules exhibit rapid tubulin turnover (Hao et al., 2011), and this localized dynamism is necessary to maintain proper 
A
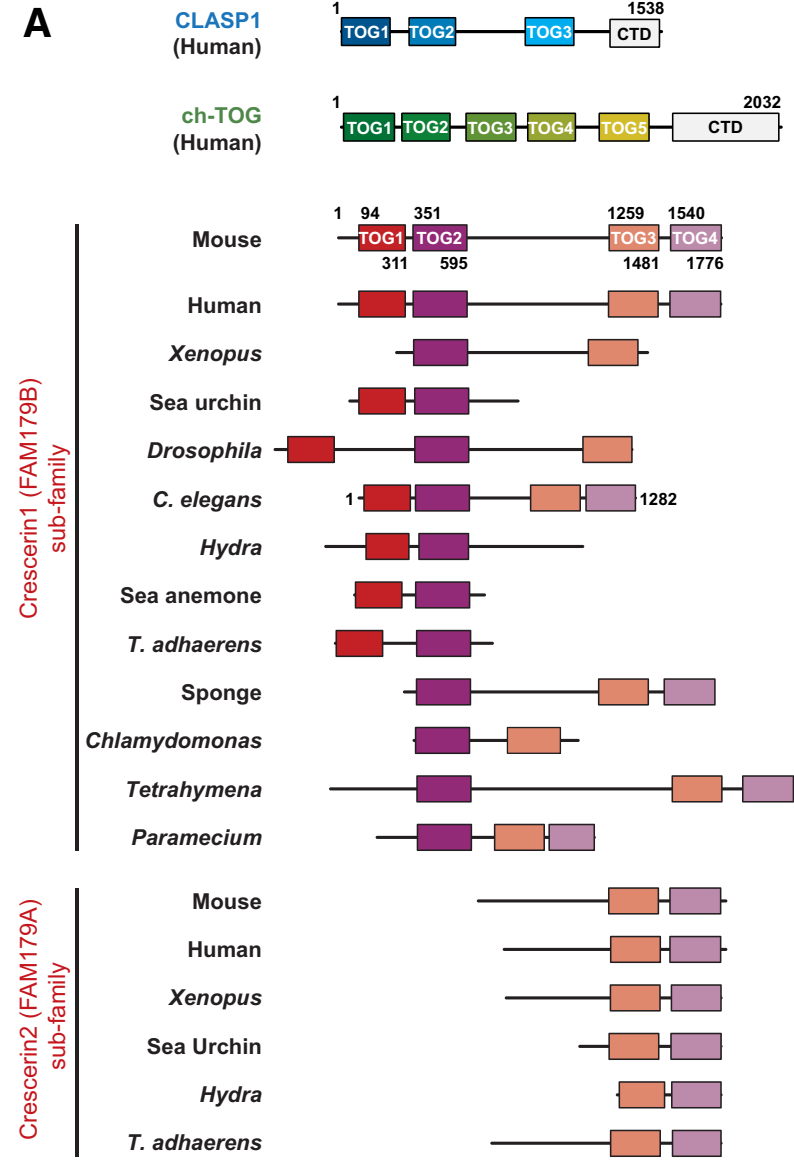

B

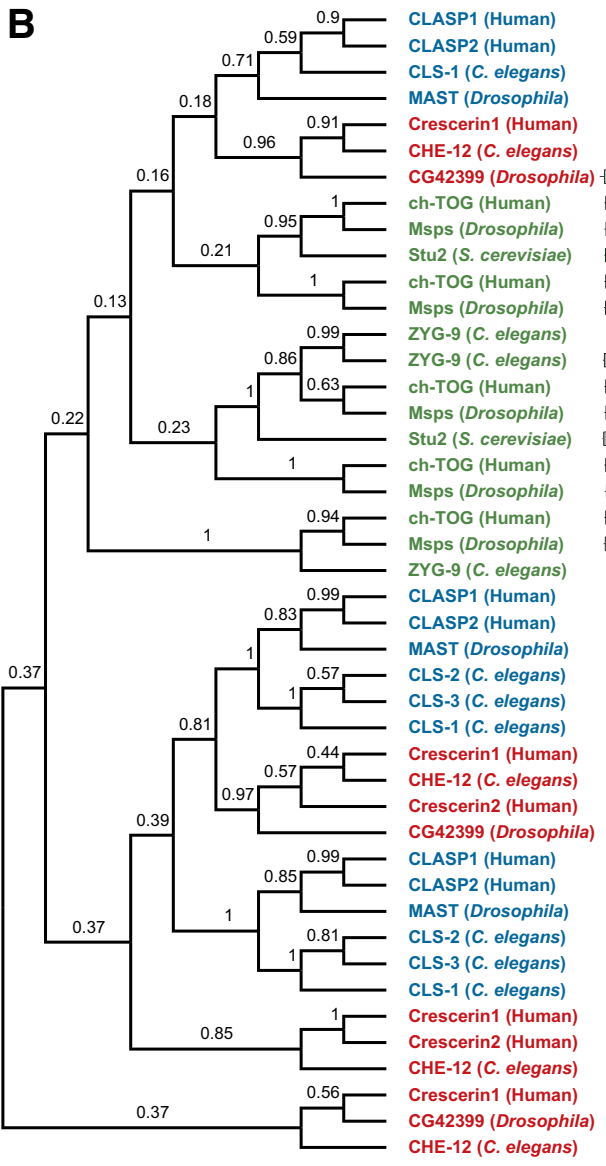

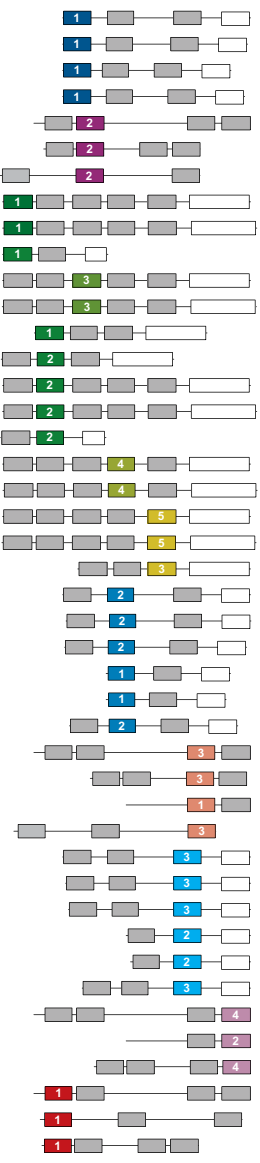

FIGURE 1: Crescerin defines a TOG domain array-containing protein family conserved across ciliated/flagellated organisms that is distinct from the ch-TOG and CLASP families. (A) Domain architecture of TOG family proteins ch-TOG, CLASP, and Crescerin. CTD, C-terminal domain in ch-TOG and CLASP. Sequence analysis of Crescerin family proteins across species reveals a variable number of TOG domains and variable lengths of inter-TOG domain linkers. In each organism, the domains are color coded according to the mouse Crescerin1 (FAM179B) TOG domain to which they are most similar in amino acid sequence. All proteins that include TOG domains similar to the $\mathrm{N}$-terminal pair of TOG domains in mouse Crescerin1 are grouped under the Crescerin1 subfamily. Some organisms, including mammals, contain a separate gene (FAM179A) with two TOG domains that are similar to the C-terminal pair of TOG domains in mouse Crescerin1. These proteins are grouped under the Crescerin2 subfamily. See Supplemental Table S1 for a list of $\mathrm{NCBI}$ sequence identifiers for all of the Crescerin homologues identified in this study. (B) Cladogram showing the relationship between TOG domains in the three TOG domain array-containing protein families, colored as in B. Branch numbers indicate bootstrap values.

axoneme structure. However, the identities and mechanisms of MTassociated proteins (MAPs) involved in regulating ciliary MT organization and dynamics are not well understood.

Whereas MT polymerization-dependent GTP hydrolysis confers the $\alpha \beta$-tubulin polymer with inherent dynamics, a host of cytoplasmic MAPs regulate MT dynamics in space and time to facilitate dynamic cellular changes as required during mitosis, polarized cell migration, and ciliogenesis. Colonic and hepatic tumor overexpressed gene (ch-TOG) and cytoskeletal linker protein 170-associated protein (CLASP) represent two distinct MAP families that promote MT polymerization and pause, respectively (Akhmanova et al., 2001; Brittle and Ohkura, 2005; Drabek et al., 2006; Sousa et al., 2007; Widlund et al., 2011). Both ch-TOG and CLASP use arrayed tubulin-binding TOG domains to regulate MT dynamics (Al-Bassam et al., 2007; Al-Bassam and Chang, 2011; Slep, 2009). The number of TOG domains in each array differs (Figure 1A), as do the architecture and tubulin-binding activity of each TOG domain. Higher eukaryotic ch-TOG family members use a pentameric TOG domain array to promote MT polymerization (Widlund et al., 2011), whereas CLASP members use a trimeric TOG domain array to stabilize MTs and promote MT pause (Al-Bassam et al., 2010; Patel et al., 2012). A TOG domain consists of six pairs of antiparallel $\alpha$-helices, termed HEAT repeats (HRs), which form an oblong, solenoid-like structure. TOG domains bind tubulin using a series of intra-HEAT loops that are discontinuous in the primary sequence but line one face of the domain in the tertiary structure. The helices and inter-HEAT loops that buttress the tubulin-binding determinants have diverged in sequence, both within a given TOG array and across the ch-TOG and CLASP families, making it challenging to identify TOG domain-containing proteins using BLAST searches. Using structure-based methodology, we previously predicted and confirmed the existence of a cryptic TOG domain (TOG2) in CLASP (Slep and Vale, 2007; Leano et al., 2013), establishing a TOG array-based paradigm for ch-TOG and CLASP family MT regulatory activity. Here we asked whether we could identify another TOG domain-containing protein family in eukaryotes based on our knowledge of TOG structural features and 
tubulin-binding determinants, and, if so, ascertain whether this protein family plays a role in regulating MTs. We identified a previously uncharacterized mammalian protein (KIAA0423; FAM179B) as a TOG domain array-containing protein that regulates cilia microtubule structure. FAM179 family proteins, which we renamed Crescerin for the Latin crescere (grow), is a large family conserved across ciliated and flagellated eukaryotes and includes Caenorhabditis elegans member CHE-12.

\section{RESULTS}

\section{Crescerin defines a conserved protein family with a predicted TOG domain array}

Given that the ch-TOG and CLASP families play central roles regulating MT dynamics, we asked whether any other conserved protein families might use a TOG domain array to regulate MT dynamics. We performed structure-based BLAST searches using individual TOG domains from ch-TOG and CLASP family members and scanned weak hits for sequence identity in the discontinuous TOG domain intra-HEAT loop determinants that bind tubulin. Searching the human proteome, we were surprised to find an uncharacterized protein, FAM179B (KIAA0423), that showed discontinuous sequence similarity to CLASP TOG1 (BLAST E-value of 1e-09). This novel protein is a member of a protein family conserved in ciliated/ flagellated eukaryotes ranging from mammals to unicellular eukaryotes, including Chlamydomonas (SSA6) and Tetrahymena, but is absent in nonciliated eukaryotes, including yeast and Dictyostelium (Figure 1A and Supplemental Table S1). We designate this protein family and its unnamed members Crescerin. The only family member characterized is the $\mathrm{C}$. elegans member $\mathrm{CHE}-12$. The single study examining $\mathrm{CHE}-12$ showed that it is expressed in a subset of amphid neurons possessing simple rod-like cilia and two phasmid sensory neurons under the transcriptional control of DAF-19, a transcription factor that controls numerous ciliary genes (Bacaj et al., 2008). Overexpressed CHE-12::green florescent protein (GFP) localizes to cilia, and mutations in che-12 impair cilia formation (Bacaj et al., 2008). Concordant with a role in cilia structure, che-12 mutant worms do not properly chemotax in response to sodium chloride (Bacaj et al., 2008).

On further investigation, we found that mammals contain two closely related Crescerin family genes, FAM179A and FAM179B (Figure 1A). Sequence analysis and secondary structure prediction of mouse FAM179B revealed four conserved domains-an $\mathrm{N}$-terminal pair and a C-terminal pair-connected by a long central linker (Figure 1A). Each of the four domains contains 12 predicted $\alpha$-helices that are predicted to form six tandem HRs (Supplemental Figures S1 and S2) with intra-HEAT loops displaying similarity to the intra-HEAT loop tubulin-binding determinants found in ch-TOG and CLASP family TOG domains. The mammalian FAM179A protein contains only two conserved TOG-like domains, which share high sequence similarity with the FAM179B C-terminal pair of TOG domains. A number of Crescerin family members from different species were found to contain fewer than four TOG domains. We classified all proteins that include TOG-like domains similar to the mammalian FAM179B TOG1 or TOG2 into the Crescerin1 subfamily and those that only contain FAM179B TOG3 and TOG4-like domains (i.e., more similar to FAM179A) into the Crescerin2 subfamily (Figure 1A and Supplemental Table S1). Dendrogram analysis of the TOG and TOG-like domains from the ch-TOG, CLASP, and Crescerin families suggests that the predicted Crescerin1 TOG-like domains TOG2, TOG3, and TOG4 are most similar to CLASP family TOG domains TOG1, TOG2, and TOG3, respectively (Figure 1B). In contrast, Crescerin1 TOG-like domain TOG1 is not closely related to
TOG domains from either the CLASP or the ch-TOG family and is the least conserved TOG domain across the Crescerin family. We sought to determine whether the Crescerin protein family represents a third TOG array-containing protein family and plays a role distinct from the ch-TOG and CLASP protein families potentially specific to cilia structure and function.

\section{Crescerin1 localizes to cilia in C. elegans amphid and phasmid neurons and mammalian cells}

The C. elegans Crescerin homologue CHE-12 was previously reported to be required for the structure and function of cilia in the amphid neurons, a subset of sensory neurons in the head (Perkins et al., 1986; Bacaj et al., 2008). Previous studies used extrachromosomal arrays to show that the che-12 promoter is active in amphid neurons and phasmid neurons, that overexpressed CHE-12 localizes to cilia, and that this localization was dependent on intraflagellar transport (IFT) particle B (Bacaj et al., 2008). To characterize endogenous $\mathrm{CHE}-12$ expression and localization, we used Cas9-triggered homologous recombination (Dickinson et al., 2013) to insert gfp into the che-12 locus, generating GFP::CHE-12 and CHE-12::GFP fusion proteins expressed under the control of all native regulatory elements (Figure 2, Supplemental Figure S3, and Supplemental Table S2). We additionally expressed mCherry under control of the gcy-5 promoter to label the ASER neuron, an amphid neuron with a single, rod-like cilium (Figure 2A). GFP::CHE-12 and CHE-12::GFP both localized to multiple sensory cilia in the head, including the bundle of parallel amphid channel cilia (Figure 2, B and C). CHE-12 was also expressed in the phasmid neurons in the tail region, where it localized along the phasmid cilia with slight enrichment near the cilia base (Figure 2D). CHE-12 GFP constructs showed punctate localization along the amphid and phasmid cilia, which may reflect association with IFT particles.

The localization of CHE-12 to amphid and phasmid neuronal cilia in C. elegans parallels the localization of endogenous Crescerin1 at the primary cilium and basal body in mammalian cells, as observed by staining mouse IMCD3 cells with an antibody we raised against mouse Crescerin1 (Figure 3A), suggesting a conserved role for the Crescerin family in cilia structure and/or function. In addition, GFPtagged, full-length mouse Crescerin 1 also localized to primary cilia in human HEK-293 cells (Figure 3, B and C). Crescerin1-GFP localized along the length of the primary cilium and at the basal body at low expression levels (Figure 3B). At high expression, Crescerin1-GFP accumulated at the basal body and distal tip of primary cilium, as well as along cytoplasmic MTs (Figure $3 \mathrm{C}$ ). Cells were fixed and stained with anti-acetylated tubulin antibody (red) to label cilia and anti-pericentrin antibody (blue) to label basal bodies and centrosomes. Localization of overexpressed Crescerin1-GFP to the distal cilia tip may imply coupling to IFT machinery. The potential for this is supported by the punctate localization we observed for CHE-12 GFP constructs along the amphid and phasmid cilia, as well as previous work that found CHE-12 localization to depend on IFT particle B (Bacaj et al., 2008). We never observed Crescerin1-GFP at the plus ends of cytoplasmic MTs, suggesting that Crescerin1 does not preferentially localize to the distal cilia tip through recognition of a GTP-MT cap.

\section{Structure of the Crescerin 1 second conserved domain reveals a bona fide TOG domain}

To determine whether the Crescerin family represents a novel TOG domain array-containing protein family, we cloned and purified all four conserved putative TOG domains from mouse Crescerin1. We obtained crystals of the second conserved domain (residues 332620 ) in space group $P 2{ }_{1} 2{ }_{1} 2$. We collected a native $x$-ray diffraction 
A
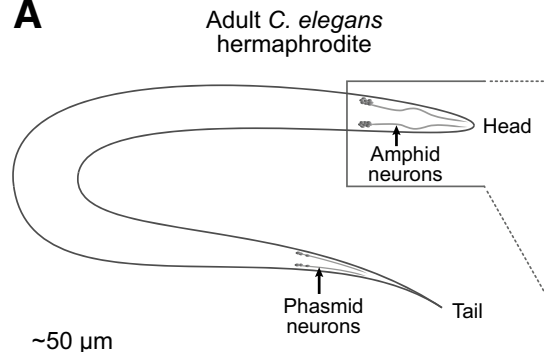

$\sim 50 \mu \mathrm{m}$

Adult $C$. elegans hermaphrodit
B

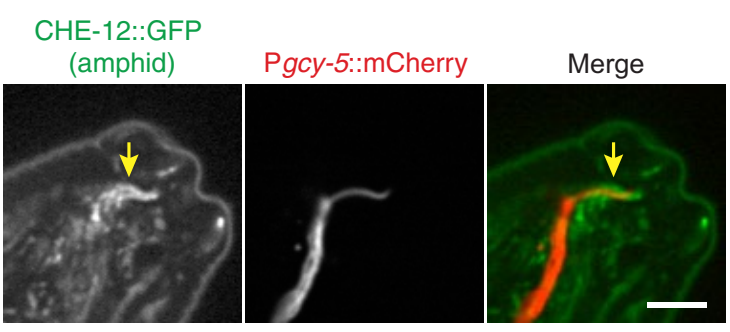

Amphid neurons

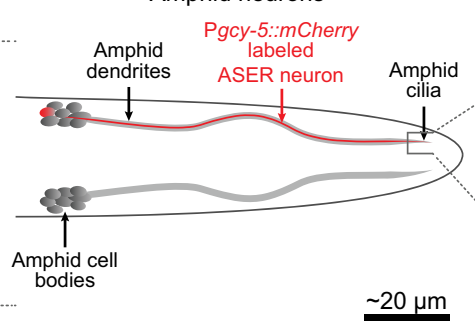

C

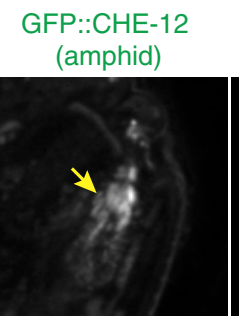

Amphid cilia

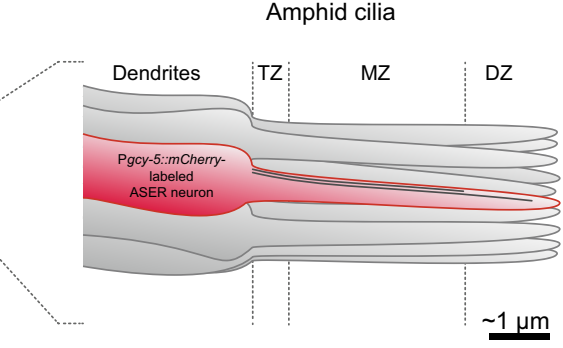

FIGURE 2: In C. elegans, the endogenously tagged Crescerin family member CHE-12 localizes to cilia in amphid and phasmid neurons. (A) Schematic showing the position of amphid and phasmid neurons in C. elegans (left). The amphid neurons each project a dendrite that extends toward the anterior end. These dendrites collectively bundle, and they terminate in cilia (right). In our studies, we labeled the ASER amphid neuron using a Pgcy-5::mCherry reporter (red). See Supplemental Figure S3 and Supplemental Table S2 for outline of modifications made to the che-12 locus.

(B, C) CHE-12::GFP (B) and GFP::CHE-12 (C) localize to amphid cilia (yellow arrow). (D) CHE-12::GFP also localizes to the cilia in phasmid neurons (PHA, PHB). Scale bars, $5 \mu \mathrm{m}(\mathrm{B}-\mathrm{D})$.

data set to $2.2-\AA \AA$ resolution and a single-wavelength anomalous dispersion data set on selenomethionine-substituted protein to 2.37- $\AA$ resolution. We solved and built the structure, refining against the native data to $2.2-\AA$ resolution, yielding $R$ and $R_{\text {free }}$ values of 0.18 and 0.24 , respectively (Supplemental Table S3). The model contains one molecule of Crescerin 1 in the asymmetric unit and encompasses residues 345-594.

The Crescerin 1 TOG2 structure reveals a bona fide TOG domain fold consisting of 12 helices that pair into six tandem HRs (A-F) and stack, forming a 60-Å oblong solenoid (Figure 4A). TOG domain HRs can be grouped into two triads: the first triad contains HRs A-C, and the second triad contains HRs D-F. These HR triads have a canonical handedness and relative positioning similar to that observed in ch-TOG family TOG domains (Al-Bassam et al., 2007; Slep and Vale, 2007; Fox et al., 2014). The HR A-C triad has a right-handed twist, whereas the HR D-F triad has a right-handed twist between repeats $D$ and $E$ but transits to a left-handed twist between repeats $\mathrm{E}$ and $\mathrm{F}$. Between the two HR triads, there is a lateral shift relative to the axis of the solenoid, a common feature found in all other TOG domain structures determined to date. The Crescerin1 TOG2 structure has inter-HR $\alpha$-helices $\alpha 2 B_{1}$, situated between HR A and HR B, also found in hCLASP1 TOG2, and $\alpha 2 \mathrm{E}_{1}$, situated between HR D and HR E, also found in the hCLASP1 TOG2 structure, as well as in ch-TOG family structures of Drosophila Msps TOG2 and TOG4. To map conserved residues across the Crescerin family onto the structure, we aligned 13 diverse species and contoured conservation at $75 \%$ identity (green) and 75\% similarity (yellow, Figure 4B and Supplemental Figure S2). The majority of conserved residues map to the intra-HEAT loops that span one face of the domain. These conserved intra-HEAT loops are similar in composition and structure to the tubulin-binding intra-HEAT loops found in ch-TOG and CLASP protein family TOG domain structures (Figure $4 \mathrm{C}$ and Supplemental
Figure S2). In addition, the electrostatic charge distribution across the first five intra-HEAT loops (HR A-E) is basic (Figure 4B) and, like other TOG domain structures determined to date, would complement the acidic nature of the $\alpha \beta$-tubulin TOG-binding site. Overall, the structure of the Crescerin 1 second conserved domain conforms to a canonical TOG architecture with conserved intra-HEAT loop determinants, homologous in composition and charge to the tubulin-binding determinants in ch-TOG and CLASP TOG domains. We thus designate this domain a TOG domain and refer to it hereafter as TOG2. This confirms our prediction that Crescerin represents a third, unique TOG domain-containing protein family.

\section{Crescerin1 TOG2 has a unique C-terminal $\beta$-hairpin that promotes domain stability}

Although Crescerin1 TOG2 has overall structural similarity to previously determined ch-TOG and CLASP family TOG domain structures, it also has architecturally unique components that differentiate it from these other TOG domain structures. One striking feature is the presence of a 21 -residue $\mathrm{C}$-terminal $\beta$-hairpin that runs alongside the second HR triad and occupies the area created by the lateral shift between the HR A-C and D-F triads. The $\beta$-hairpin forms multiple hydrophobic interactions and hydrogen bonds with conserved residues in the $\alpha 2 \mathrm{C}^{\prime}, \alpha 2 \mathrm{D}^{\prime}$, and $\alpha 2 \mathrm{E}^{\prime}$ helices (we designate the helices of each HR as X and $X^{\prime}$, as in Slep and Vale, 2007, with the numeral 2 indicating TOG2). An invariant asparagine (N491) in $\alpha 2 D^{\prime}$ forms a hydrogen bond with a conserved tyrosine residue (Y588) on the $\beta 2^{\prime}$ strand of the $\beta$-hairpin. The $\beta$-hairpin is further locked alongside the TOG domain by a network of hydrogen bonds formed between the A589 backbone amide and a conserved glutamate side chain (E531) in $\alpha 2 E^{\prime}$, as well as the T576 backbone carbonyl group and a conserved arginine side chain (R571) in helix $\alpha 2 \mathrm{~F}^{\prime}$ (Figure 4D). Commensurate with a stable, bona fide interaction 


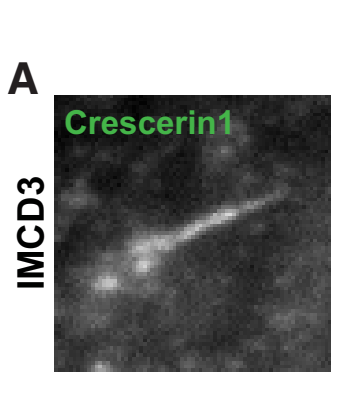

Acetylated
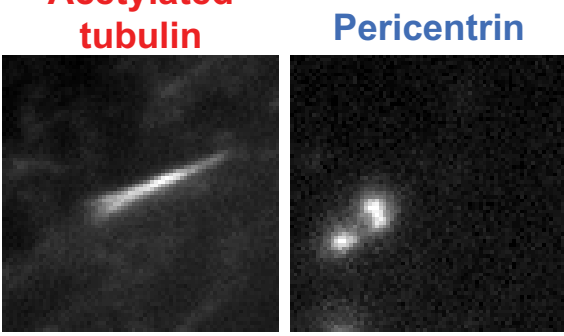

B
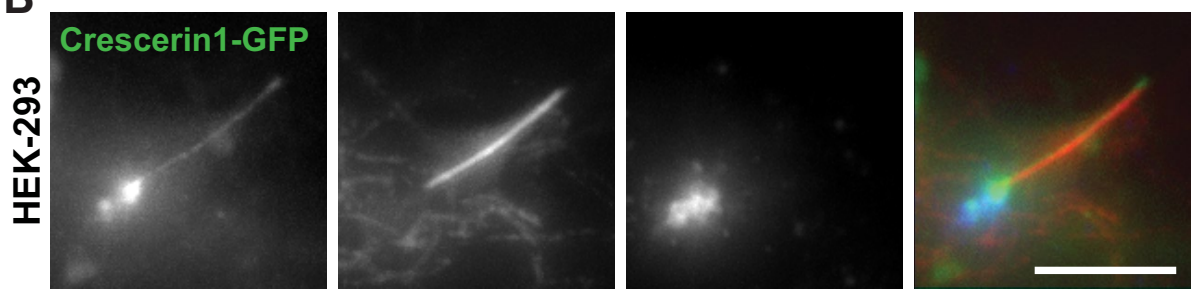

C
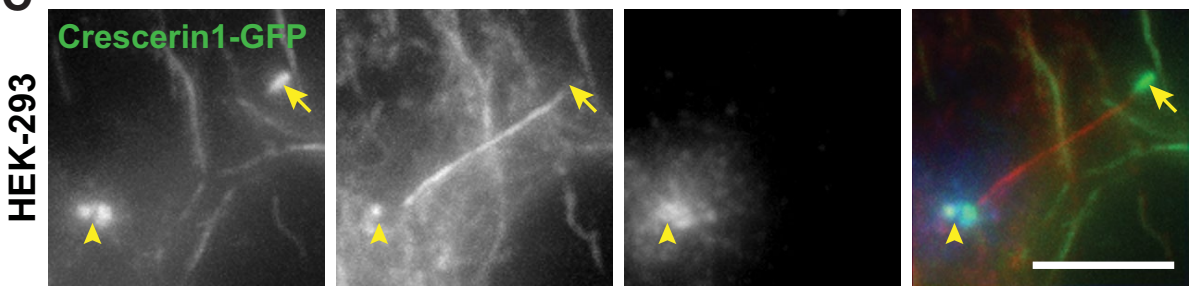

FIGURE 3: Crescerin1 localizes to the primary cilium in mammalian cells. (A) Antibody staining against endogenous Crescerin1 in IMCD3 cells shows localization to the primary cilium and basal body. (B) GFP-tagged mouse Crescerin1 localizes along the primary cilium and to the basal body in HEK-293 cells. (C) At high expression levels, GFP-tagged Crescerin1 accumulates at the basal body (yellow arrowhead) and distal tip (yellow arrow) of the primary cilium, as well as along the lattice of cytoplasmic microtubules. Primary cilia are labeled using antibody against acetylated tubulin (red), and basal bodies are labeled using antibody against pericentrin (blue). Scale bars, $5 \mu \mathrm{m}$.

between the $\beta$-hairpin and the central HRs, the $\beta$-hairpin has comparatively low $B$-factors that parallel those of the HRs with which it interacts. To assess further the contribution of the C-terminal $\beta$-hairpin to domain stability, we created a shorter TOG2 construct lacking the $\beta$-hairpin (TOG2- $\Delta \beta H P$, residues $332-576$ ) and performed secondary structure analysis by circular dichroism (CD). Although the overall $\alpha$-helical signature was similar for both constructs (Figure $4 \mathrm{E}$ ), the TOG2- $\Delta \beta \mathrm{HP}$ construct had a lower melting temperature $\left(T_{\mathrm{m}}=45.5^{\circ} \mathrm{C}\right)$ than the TOG2 construct containing the $\beta$-hairpin (residues $332-620 ; T_{m}=57.5^{\circ} \mathrm{C}$ ), indicating that the $\beta$-hairpin promotes TOG2 domain stability (Figure 4F). Secondary structure elements that flank TOG domains have been shown to stabilize other TOG domains (Leano et al., 2013). Crescerin family sequence alignments and secondary structure prediction analysis on the regions flanking TOG domains 1,3 , and 4 did not show conservation or similar predicted secondary structure, suggesting that the TOG2 C-terminal $\beta$-hairpin is a unique stabilizing feature for Crescerin 1 TOG2. Whether this $\beta$-hairpin plays additional functional roles remains to be determined.

\section{The Crescerin1 TOG2 tubulin-binding surface is similar to Stu2 TOG1}

TOG domain structures from the ch-TOG and CLASP families reveal distinct structural variations that predict diversified interactions with $\alpha \beta$-tubulin. To determine the TOG structures to which Crescerin 1 TOG2 is most similar, we performed pairwise structural

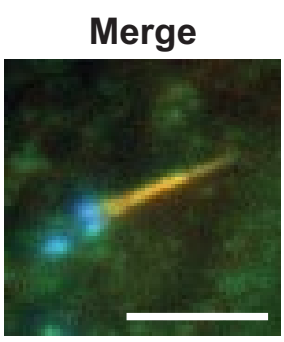

alignments using the DALI server (Holm and Rosenström, 2010), aligning the first HR triads as a point of reference, since this region displays the highest conservation across TOG domains (Figure 5A). The Crescerin1 TOG2 intra-HEAT loop surface assumes a straight conformation that closely resembles Stu2 TOG1 and contrasts with the bent architectures observed in ch-TOG TOG4, MAST TOG1, and hCLASP1 TOG2 (Figure 5A, red arrows; Slep and Vale, 2007; Ayaz et al., 2012; De La Mora-Rey et al., 2013; Leano et al., 2013; Fox et al., 2014). To gain insight into whether Crescerin1 TOG2 could form contacts with tubulin as observed in the Stu2 TOG1- $\alpha \beta$-tubulin structure, we docked Crescerin1 TOG2 on the tubulin heterodimer by structurally aligning Crescerin1 TOG2 HRs A-C (first HR triad) and HRs D-F (second HR triad) to the coordinates of Stu2 TOG1 in the Stu2 TOG1-tubulin complex (Ayaz et al., 2012; Figure 5, B-D and E-G, respectively). Many of the Stu2 TOG1 tubulin-binding residues are conserved and similarly positioned in Crescerin1 TOG2 (Figure 5, B-G). Prime $\beta$ tubulin binding determinants reside in Stu2 TOG1 HR A, including W23 and K24. Although electron density for the Crescerin1 TOG2 HR-A loop was poor and precluded modeling of the homologous residues $\mathrm{Y} 364$ and $\mathrm{K} 365$, the HR A helices are similarly positioned, and we anticipate that Y364 and K365 form homologous contacts with $\beta$ tubulin T107 and S400, respectively (T109 and $\mathrm{G} 410$ in mammalian $\beta$-tubulin; Figure 5C). In the Crescerin1 TOG2 HR-B and HR-C intra-HEAT loops, N406 and K447 occupy positions analogous to Stu2 N68 and R116 respectively, both of which interact with yeast $\beta$-tubulin Y106 (Y108 in mammalian $\beta$-tubulin; Figure 5D). In Crescerin1 TOG2 HR-D, K481 and R484 are positioned similarly to Stu2 K151 and K154, which interact with $\beta$-tubulin E158 and E157, respectively (E160 and E159 in mammalian $\beta$-tubulin; Figure 5F). Similarly, the highly conserved R526 in Crescerin1 TOG2 HR-E is positioned analogously to Stu2 R200, which interacts with $\alpha$-tubulin V410, and E415 (V409 and E414 in mammalian $\alpha$-tubulin; Figure 5G). The composition and conformational similarity between Stu2 TOG1 and Crescerin1 TOG2 intra-HEAT loops suggests that Crescerin1 TOG2 might be involved in binding free $\alpha \beta$-tubulin and/or MTs to regulate MT dynamics.

\section{Crescerin1 TOG domains promote MT polymerization in vitro}

To determine whether Crescerin might regulate the MT cytoskeleton, we first tested whether Crescerin1 TOG domains could influence MT polymerization in vitro. We mixed different purified TOG domains (Figure 6A) with tubulin and monitored tubulin polymerization at $37^{\circ} \mathrm{C}$ using a light scattering assay. The intrinsic polymerization activity of tubulin in the absence of other proteins is reflected as a characteristic sigmoidal increase in light scattering signal with an initial lag phase of $\sim 500 \mathrm{~s}$ (Figure 6B, black curve). Addition of TOG1 or TOG3 to the reaction mixture did not affect the MT polymerization rate. In contrast, addition of either TOG2 or 
A

A
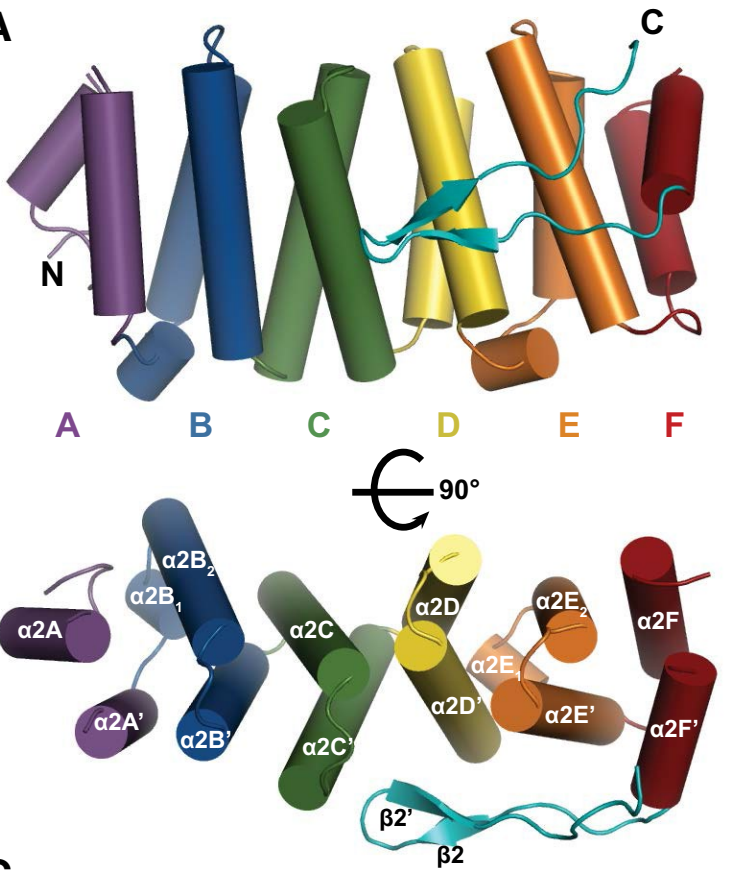

C

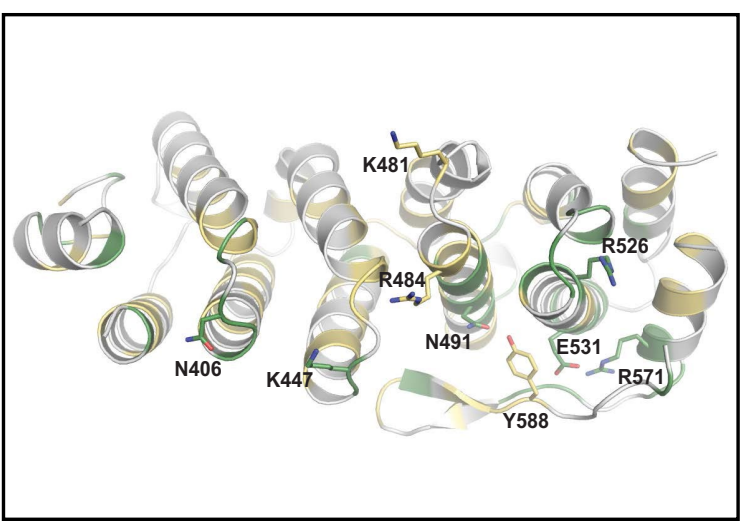

E

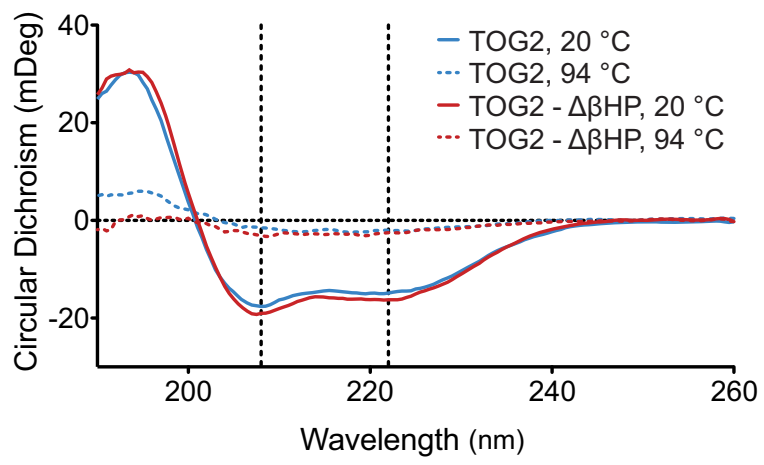

B
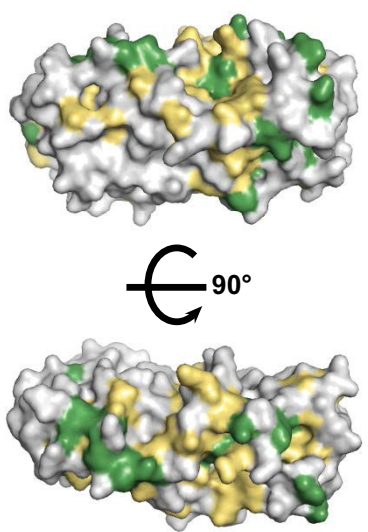

$75 \%$ Identity

$75 \%$ Similarity

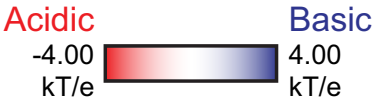

D

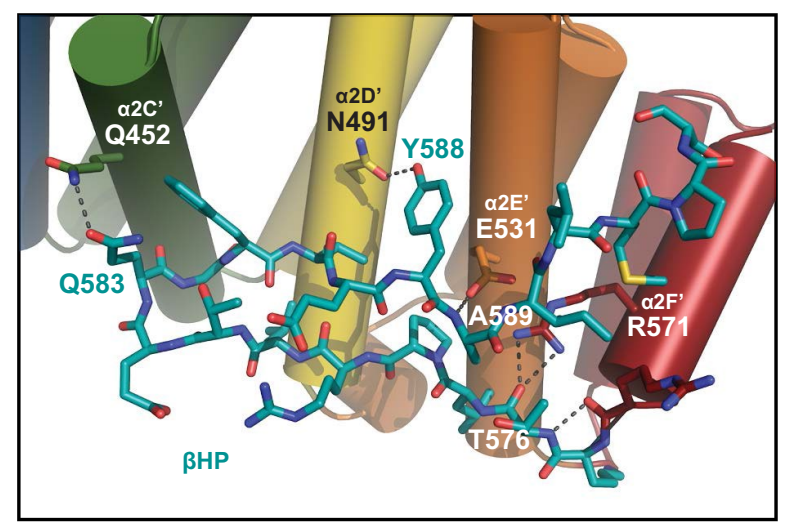

F

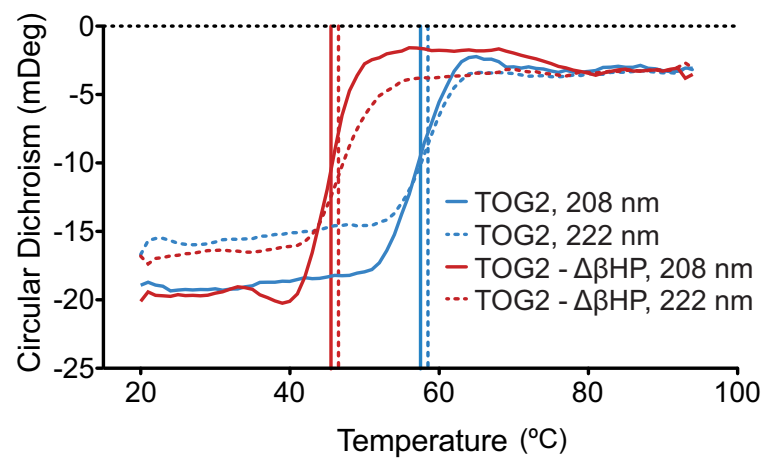

FIGURE 4: The Crescerin1 second conserved domain is a bona fide TOG domain with unique features. (A) Crystal structure of Crescerin1 TOG2. Each HR (A-F) and the C-terminal $\beta$-hairpin are shown in a different color. See Supplemental Table S3 for crystallographic data, phasing, and refinement statistics. (B) Crescerin1 TOG2 shown in surface representation, oriented as in A, with cross-species conservation (left) or electrostatic charge distribution (right) mapped on the surface. See Supplemental Figure S2 for multiple sequence alignment of Crescerin TOG2 showing conservation across species. (C) Crescerin1 TOG2 conserved residues (colored as in B) map to the tubulin-binding intra-HEAT loops and to the C-terminal $\beta$-hairpin-HR interface. (D) Expanded view of the Crescerin1 TOG2 C-terminal $\beta$-hairpin-HR interface. (E, F) Circular dichroism spectra (E) of Crescerin1 TOG2 (blue) and TOG2- $\Delta \beta H P\left(\right.$ red) at $20^{\circ} \mathrm{C}$ (solid lines) and $94^{\circ} \mathrm{C}$ (dotted lines) and thermal melt curves (F) monitored using 208-nm (solid lines) and 222-nm (dotted lines) light. 
A
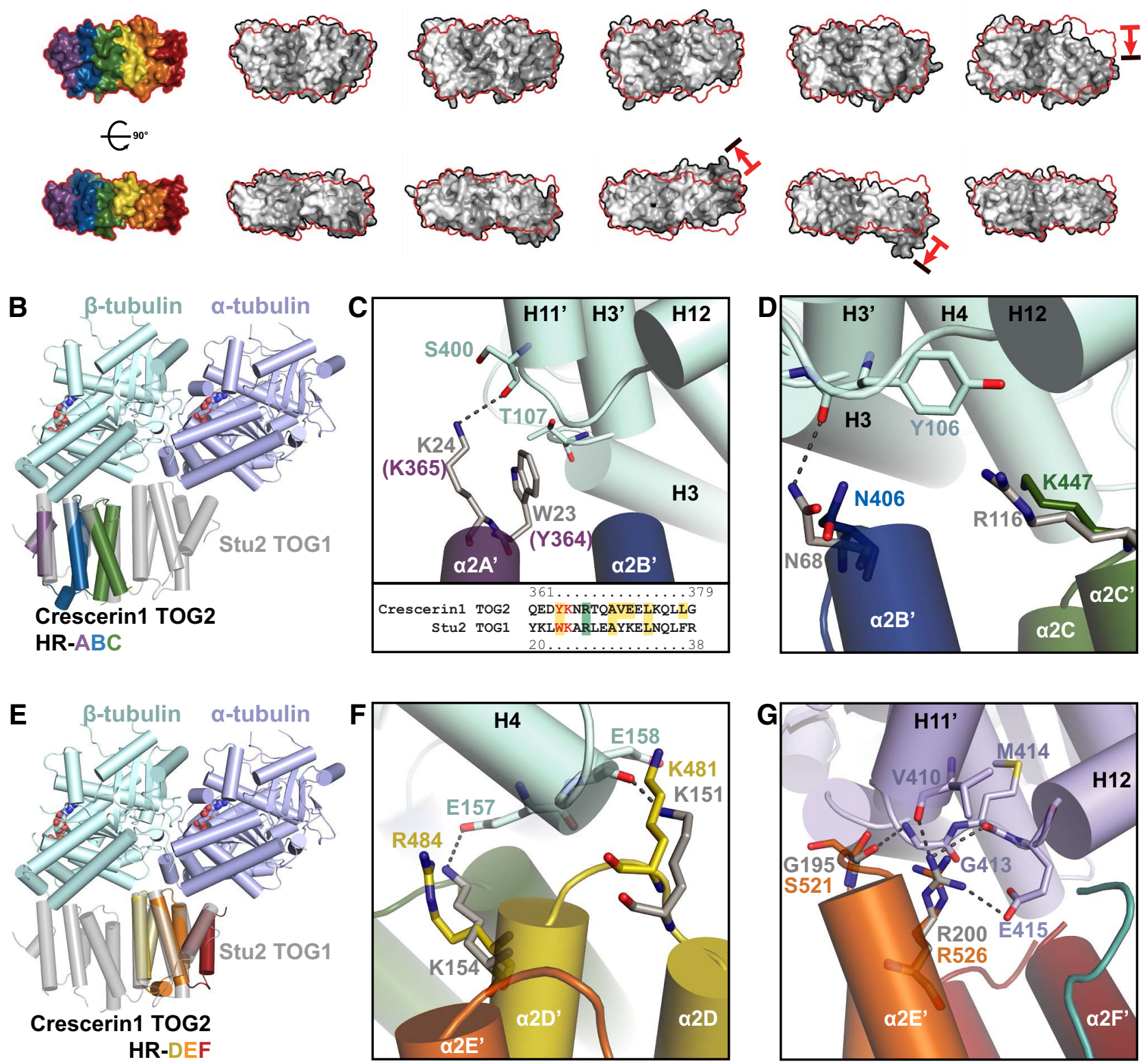

FIGURE 5: The Crescerin1 TOG2 tubulin-binding surface is similar to that of Stu2 TOG1. (A) Comparison of Crescerin1 TOG2 structure (rainbow/red outline) with Stu2 TOG1 (4FFB), Stu2 TOG2 (2OK1), ch-TOG4 (4OMI), MAST TOG1 (4G3A), and hCLASP1 TOG2 (4K92) structures by aligning the first HR triad. Red arrows highlight major structural differences. (B-D) Alignment of the Crescerin1 TOG2 first HR triad to the Stu2 TOG1- $\alpha \beta$-tubulin complex (4FFB). (C) Stu2 TOG1 HR A-loop residues W23 and K24 interact with T107 and S400 of $\beta$-tubulin. Although residues in the Crescerin1 TOG2 HR A loop could not be modeled due to weak electron density, we anticipate that the homologous Crescerin 1 residues Y364 and K365 (see alignment below) form similar interactions with $\beta$-tubulin. (D) Crescerin1 TOG2 residues N406 and K447 are positioned similar to Stu2 TOG1 residues N68 and R116, which interact with $\beta$-tubulin Y106. (E-G) Alignment of the Crescerin1 TOG2 second HR triad to the Stu2 TOG1- $\alpha \beta$-tubulin complex (4FFB).

(F) Crescerin1 residues K481 and R484 occupy similar positions as Stu2 TOG1 K151 and K154, which form salt bridges with $\beta$-tubulin E158 and E157, respectively. (G) Crescerin1 TOG2 HR-E residues S521 and R526 occupy similar positions as Stu2 TOG1 G195 and R200, which interact with $\alpha$-tubulin V410, G413, M414, and E415.

TOG4 dramatically increased the rate of MT polymerization. To determine whether TOG2 and TOG4 potentiated tubulin polymerization using canonical tubulin-binding determinants, we mutated a conserved aromatic residue in the HR A intra-HEAT loop in each TOG domain to glutamate (Y364E in TOG2 and F1559E in TOG4). Homologous mutations in other TOG domains have been shown to abrogate tubulin binding without affecting the TOG domain fold
(Slep and Vale, 2007; Leano et al., 2013). We performed CD spectrum analysis on these constructs and confirmed that Y364E and F1559E mutations in TOG2 and TOG4, respectively, did not affect the $\alpha$-helical fold and thermal stability of these domains (Supplemental Figure S4). The Y364E and F1559E mutations abrogated the ability of Crescerin 1 TOG 2 and TOG4, respectively, to accelerate tubulin polymerization, suggesting that both TOG2 and TOG4 use 


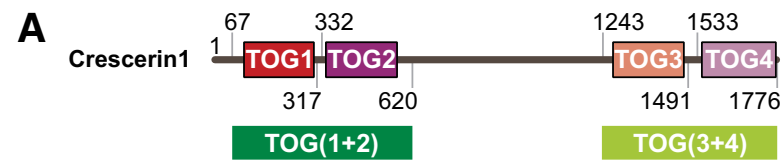

B

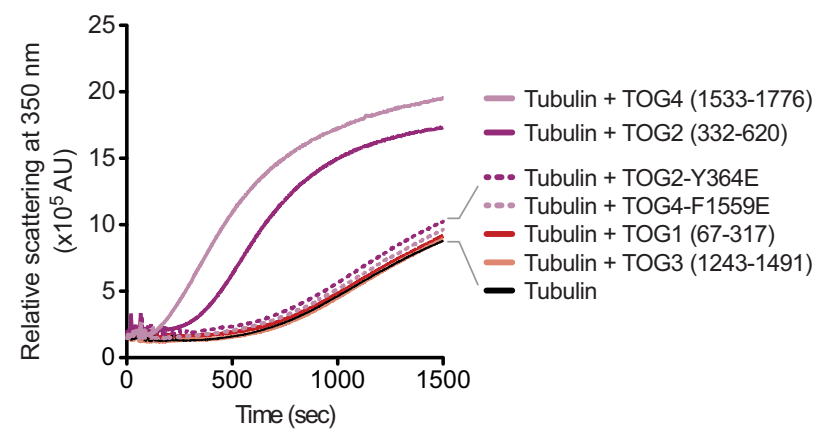

C

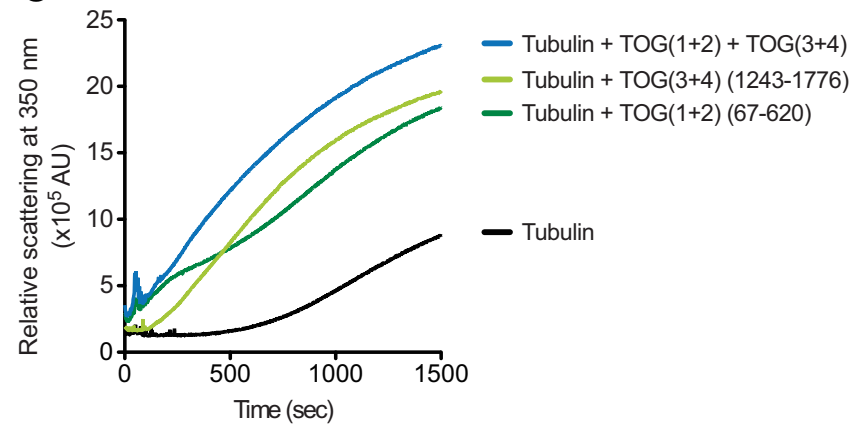

FIGURE 6: Crescerin1 TOG domains affect MT polymerization in vitro. (A) Schematic of Crescerin1 TOG domain-containing fragments used for in vitro MT polymerization assays. (B) In vitro MT polymerization assays in the absence (black) or presence of purified Crescerin 1 single-TOG domains. TOG2- and TOG4-dependent MT polymerizing activity was lost when key tubulin-binding HR A-loop residues Y364 (TOG2) and F1559 (TOG4) were mutated to glutamate (dotted lines). Addition of TOG1 (red) or TOG3 (orange) did not affect MT polymerization kinetics. See Supplemental Figure S4 for CD analysis of single-TOG domain constructs used in B. (C) Larger constructs containing tandem TOG domains 1 and 2 (dark green) or 3 and 4 (light green) increased MT polymerization kinetics.

canonical tubulin-binding determinants to promote tubulin polymerization. Addition of larger fragments containing pairs of TOG domains (TOG(1+2), amino acids 67-620; and TOG(3+4), amino acids 1243-1776) resulted in distinct reproducible scattering curves with short lag times (Figure 6C). These data suggest that Crescerin family TOG domains have differential tubulin-binding activity and might function collectively to regulate MT dynamics.

\section{Crescerin1 C-terminal TOG domains promote MT lattice binding}

We previously noticed that overexpression of full-length Crescerin1 in both HEK-293 and IMCD3 cells caused cytoplasmic MT bundling, with Crescerin 1 localizing along the MT lattice (Figure $3 \mathrm{C}$ and Supplemental Figure S5, A and B). Crescerin1 MT colocalization did not correlate with increased MT acetylation, a posttranslational modification common to ciliary MTs. This suggests that Crescerin1 has an inherent ability to associate with MTs. Although Crescerin1's cytoplasmic MT colocalization is unlikely to be biologically relevant, since it is only observed upon overexpression, we took advantage of this phenotype as an assay to probe the differential contribution of Crescerin1 TOG domains to MT lattice-association activity. We compared cells with similar Crescerin1-GFP expression levels and classified MT lattice binding as strong, weak, or no lattice binding when Crescerin1-GFP colocalized with MTs completely, partially, or was diffuse in the cytoplasm, respectively (Figure 7, A-C). Fulllength Crescerin 1 was found to strongly colocalize with MTs in 72.3 $\pm 2.4 \%$ of transfected cells (Figure 7D). Mutating the intra-HEAT HR A loop tubulin-binding region in TOG1 and TOG2 (P109E and Y364E, respectively) did not appreciably alter MT-association activity. In contrast, mutating the intra-HEAT HR A-loop tubulin-binding region in TOG3 and TOG4 to glutamate (W1274E and F1559E, respectively) completely abrogated MT association. An N-terminal Crescerin1 truncation construct containing the central linker and TOG domains 3 and 4 (L-TOG-34, residues 577-1776) also had robust MT-association activity (Figure 7D). Crescerin1 L-TOG-34 MT association was fully ablated when the TOG4 HR A loop was mutated. A smaller Crescerin $1 \mathrm{~N}$-terminal truncation construct containing only a small portion of the central linker and TOG domains 3 and 4 ( $L_{c}$-TOG-34, residues 1119-1776) showed minimal MT association, which was dependent on TOG3 and TOG4 HR A-loop tubulin-binding determinants. These data suggest that Crescerin1 TOG3 and TOG4 use canonical TOG domain intra-HEAT loop determinants to engage the straight tubulin conformation found in MTs and function synergistically with determinants in the central linker region to bind MTs. This also suggests that whereas the Crescerin $1 \mathrm{~N}$-terminal region encompassing TOG domains 1 and 2 has MT polymerization activity, it does not contribute dramatically to MT lattice association in our cellular overexpression assay, a characteristic that is shared with the polarized pentameric TOG domain array of the ch-TOG protein family (Currie et al., 2011, Fox et al., 2014).

\section{The MT-regulatory function of CHE-12 is necessary for proper cilia development in C. elegans}

We next sought to investigate how Crescerin affects cilia structure and function. Because it is difficult to exercise precise temporal control on Crescerin expression in mammalian cells to match endogenous levels at different cell cycle stages, we turned to $C$. elegans as a model system for these experiments, where we have the tools perform structure/function experiments by modifying the endogenous che-12 locus. As discussed earlier, the $C$. elegans Crescerin family member $\mathrm{CHE}-12$ localizes to amphid and phasmid sensory cilia (Bacaj et al., 2008; our results). Two che-12 mutant alleles, mn389 and mn399, were previously shown to cause shortened amphid cilia (Bacaj et al., 2008). However, these two mutations truncate the CHE-12 protein after the second TOG domain and may not be molecular null mutations. To determine the effect of a complete loss of CHE-12 function, we used Cas9-triggered homologous recombination to delete the entire che-12 coding sequence and replace it with gfp (Supplemental Figure S3). As expected, the resulting $\Delta$ che-12 animals expressed GFP primarily in the amphid and phasmid sensory neurons, with GFP signal observed in the cell body and dendrite, overlapping with Pgcy-5::mCherry expression in the ASER neuron (Supplemental Figure S5C). To probe the role of $\mathrm{CHE}-12$ in cilia structure, we first scored cilium length in ASER neurons using the Pgcy-5::mCherry reporter to visualize the ASER dendrite and cilium (Figure 8, A and B). ASER cilia in the wild-type (WT) N2 strain were $7.0 \pm 1.7 \mu \mathrm{m}$ in length. Strikingly, cilia in the $\Delta$ che-12 strain were dramatically shorter, with a length of $3.2 \pm 0.6 \mu \mathrm{m}$. The phenotype of the che-12 alleles mn389 and mn399 that truncate CHE-12 after TOG2 are milder, with a $\sim 30 \%$ decrease in cilia length for the che-12(mn399) mutant compared with WT (Bacaj et al., 2008). 
A

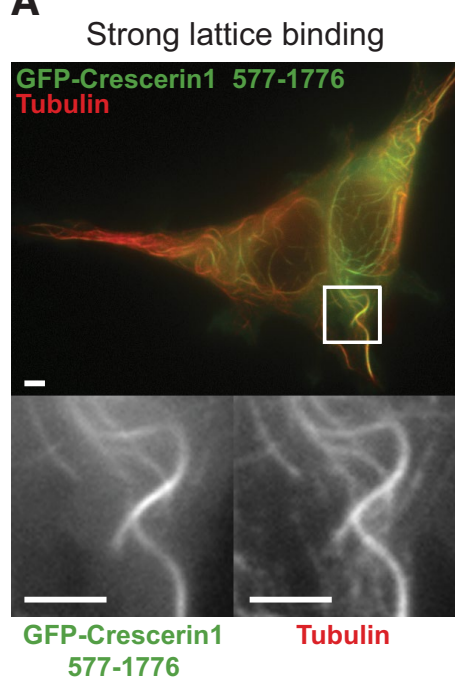

D

\section{Crescerin1}

FL (1-1776)

FL-TOG-1'2'34

FL-TOG-123'4

L-TOG-34 (577-1776)

L-TOG-3'4

L-TOG-34

L-TOG-3'4'
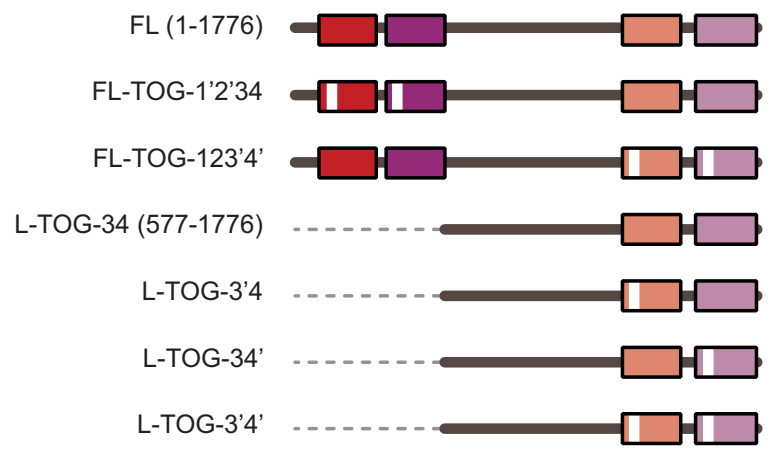

L-TOG-3 (577-1491)

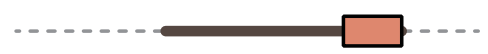

L (577-1242)

L -TOG-34 (1119-1776)

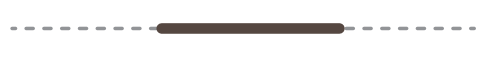

$L_{c}-T_{O G}-3^{\prime} 4$
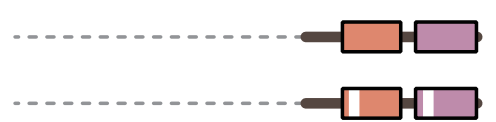

B

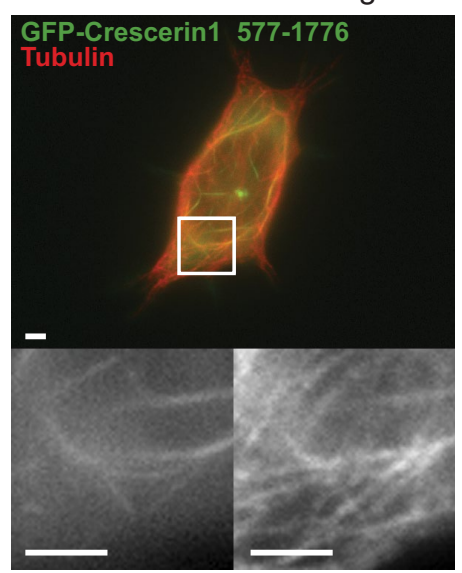

Tubulin 577-1776
C

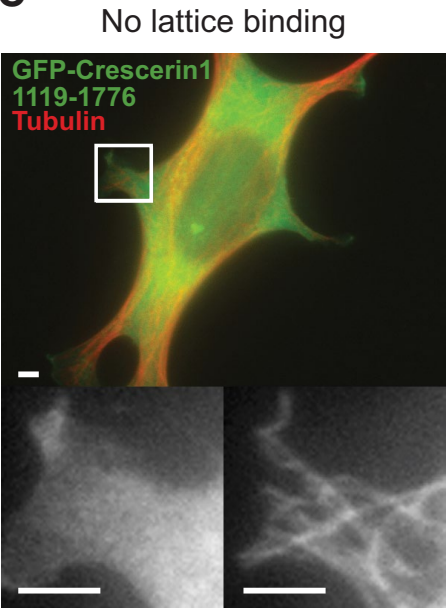

GFP-Crescerin1 1119-1776
Tubulin

Tubulin
Frequency of observed lattice binding (\%)

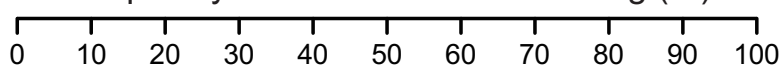

\begin{tabular}{|c|c|c|}
\hline $72.3 \pm 2.4$ & \multicolumn{2}{|c|}{ 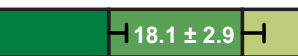 } \\
\hline $58.4 \pm 1.3$ & H $21.2 \pm 2.9$ & $20.4 \pm 1.5$ \\
\hline & & $100.0 \pm 0.0$ \\
\hline
\end{tabular}

\begin{tabular}{|l|r|r|}
\hline $56.9 \pm 4.3$ & $\begin{array}{r}17.2 \\
\pm 3.6\end{array}$ & \multicolumn{1}{l}{$25.8 \pm 7.6$} \\
\hline
\end{tabular}

\begin{tabular}{|l|l|ll|}
\hline $56.6 \pm 2.2$ & $-17.5 \pm 2.2$ & & $25.8 \pm 0.5$ \\
\hline
\end{tabular}

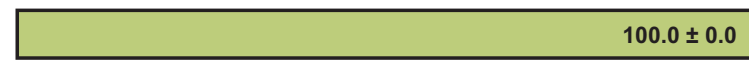

$100.0 \pm 0.0$

$100.0 \pm 0.0$

$100.0 \pm 0.0$

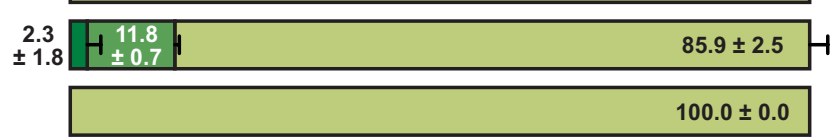

Strong binding $\square$ Weak binding $\square$ No binding

FIGURE 7: Crescerin1 C-terminal TOG domains have MT lattice-binding activity. (A-C) Examples of strong (A), weak (B), and no (C) MT lattice association by different Crescerin1 fragments. (D) Summary of MT lattice-binding observations in HEK-293 cells indicates the importance of the Crescerin1 central linker and C-terminal TOG domain pair for MT lattice association. White vertical lines on select TOG domains indicate an HR A-loop mutation that ablates tubulin-binding activity (TOG1:P109E; TOG2:Y364E; TOG3:W1274E; TOG4:F1559E).

The more severe $54 \%$ decrease in cilia length that we observed in the $\Delta$ che- 12 animals suggests that the $\mathrm{mn} 399$ strain may retain partial CHE-12 function and highlights the efficacy of Cas9-triggered homologous recombination to generate complete gene deletions with full loss-of-function phenotypes.

We also measured cilia length in the GFP-tagged CHE-12 strains. Both CHE-12::GFP and GFP::CHE-12 knock-in animals had cilia that were slightly shorter than WT $(6.0 \pm 1.5$ and $5.9 \pm 1.5 \mu \mathrm{m}$, respectively), suggesting that the terminal tag might have interfered slightly with CHE-12 function, although not as dramatically as the che-12 deletion. A control strain, expressing a labeled nonneuronal protein
(tagRFP::PH) in the same genetic background and containing the same unc-119 rescue marker used in all Cas9-mediated recombination experiments in this study had amphid cilia lengths that did not deviate statistically from WT (Figure $8, A$ and B).

We next asked whether $\mathrm{CHE}-12$ uses canonical tubulin-binding determinants across its tetra-TOG domain array to promote proper cilia length. To answer this, we mutated a conserved hydrophobic residue on the predicted tubulin-binding surface of each TOG domain HR A intra-HEAT loop (F34, A281, W815, W1073) to glutamate (denoted TOG-1'2'3'4') as we did for Crescerin1 in vitro and cellular studies. We used Cas9-triggered homologous recombination to 
A

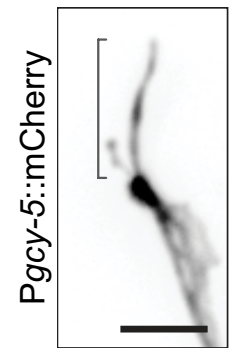

Wild-type

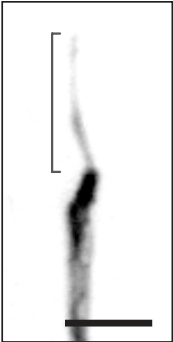

tagRFP::PH

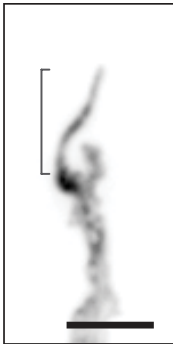

CHE-12::GFP

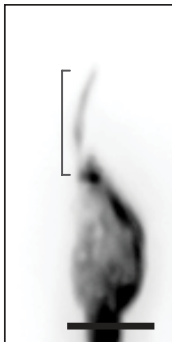

GFP::CHE-12

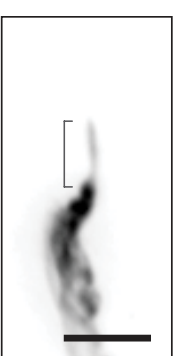

$\Delta$ che-12
B

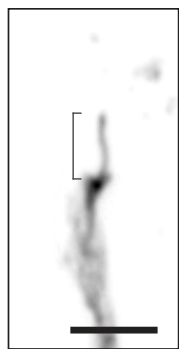

GFP::CHE-12 TOG-1'2'3'4'

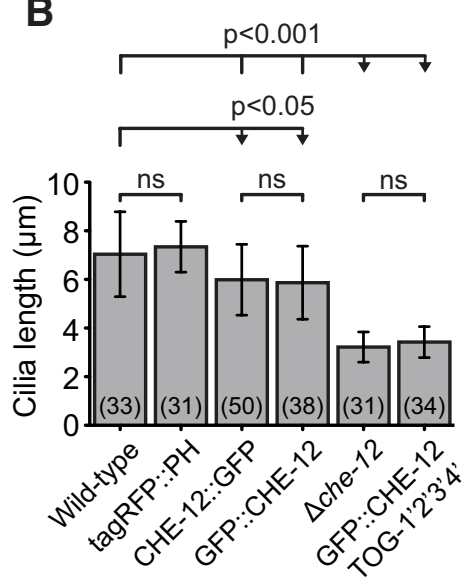

C

Proximal

Distal
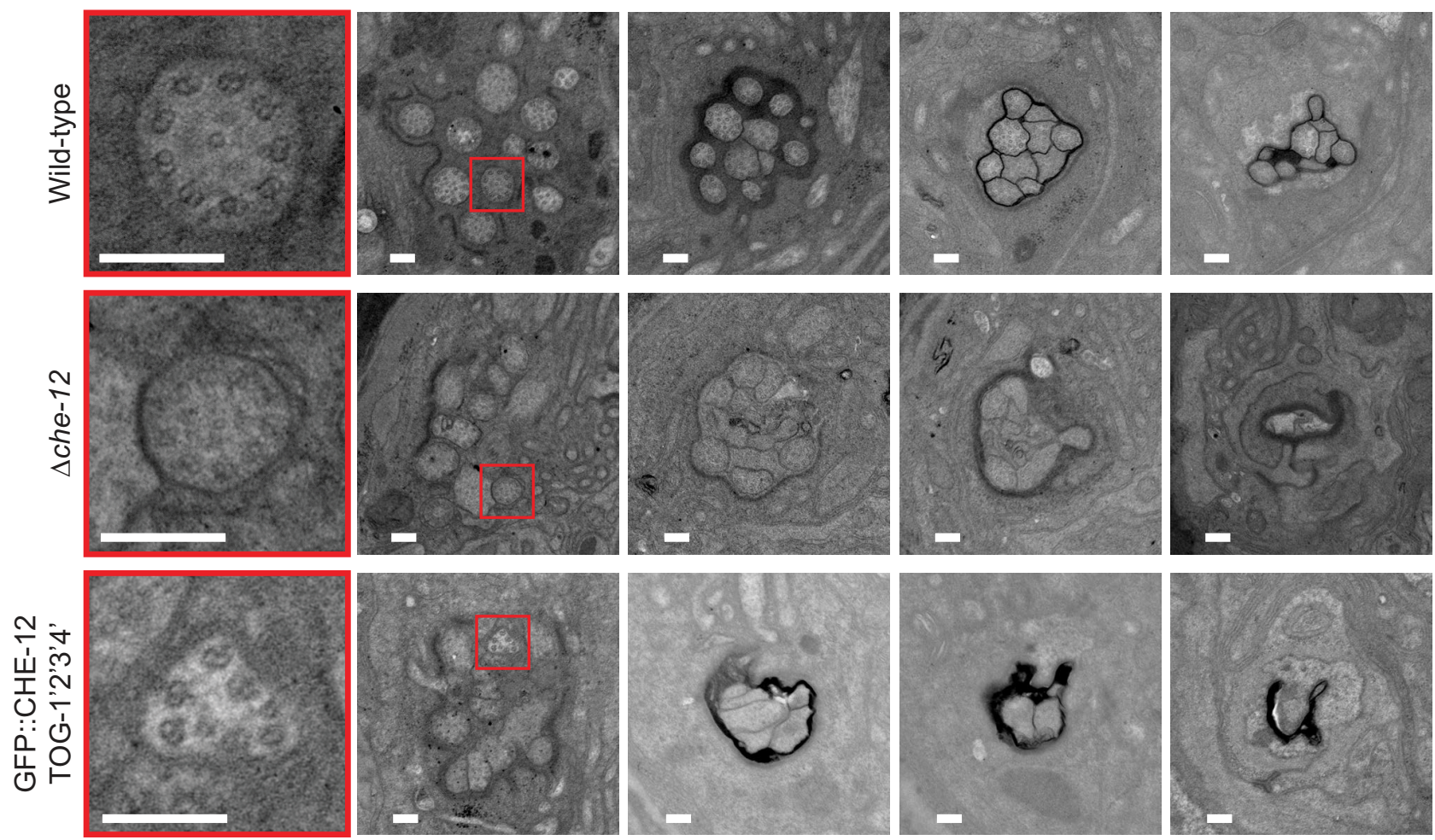

FIGURE 8: Deleting che-12 or mutating tubulin-binding residues in CHE-12 TOG domains results in shorter and disorganized cilia in C. elegans amphid neurons. (A) Images of the Pgcy-5::mCherry-labeled ASER dendrite with sensory cilium (bracket) from WT and mutant strains. Scale bars, $5 \mu \mathrm{m}$. (B) ASER cilium length for strains shown in A. Values in parentheses indicate number of cilia measured/strain; error bars indicate SD. (C) Cross-sectional transmission electron micrographs of amphid sensilla in WT, $\Delta$ che-12, and GFP::CHE-12 TOG-1' $2^{\prime} 3^{\prime} 4^{\prime}$ strains. Left, expanded views of smaller, red-boxed regions on the right. Scale bars, $200 \mathrm{~nm}$.

make these mutations at the native che-12 locus, and at the same time we introduced a GFP tag (Supplemental Figure S3). Mutant GFP::CHE-12 TOG-1'2'3'4' was expressed and localized to the cilia, indicating that tubulin-binding via TOG domains is not necessary for CHE-12 cilia localization (Supplemental Figure S5D). Strikingly, the GFP::CHE-12 TOG-1'2'3'4' mutant had amphid sensory cilia of dramatically reduced length $(3.4 \pm 0.6 \mu \mathrm{m}$ compared with WT $7.0 \pm 1.7 \mu \mathrm{m}$ ), phenocopying the che-12 knockout (3.2 $\pm 0.6 \mu \mathrm{m}$; Figure $8, \mathrm{~A}$ and $\mathrm{B})$. These results strongly suggest that $\mathrm{CHE}-12$ promotes proper cilia structure and length through mechanistic use of its TOG array's tubulin-binding activity.
Previous electron microscopy work examining the mutant strain che-12(mn389) found ultrastructural defects in amphid cilia structure and the organization of MTs in these cilia (Bacaj et al., 2008). To determine what effect complete loss of $\mathrm{CHE}-12$ function has on cilia ultrastructure, we examined cilia in WT, $\Delta$ che-12, and GFP::CHE-12 TOG-1'2'3'4' mutant worms using electron microscopy. In WT worms, the proximal sections of each of the 10 amphid channel cilia run parallel to each other, forming a well-organized bundle, with most cilia exhibiting the canonical ring of nine doublet MTs surrounding a variable number of central singlet MTs (Figure $8 \mathrm{C}$ ). These bundled cilia become smaller and more tightly packed toward their 
distal end, but the ring of nine doublet MTs is still evident. In contrast, $\Delta$ che-12 and GFP::CHE-12 TOG-1'2'3'4' mutant worm cilia had poorly defined MT architecture at their proximal ends and no apparent MTs toward their distal ends. Cilia within the bundles appeared disorganized and did not run strictly parallel to each other as in WT, which could account for decreased MT visibility on EM cross sections.

\section{CHE-12 is required for proper chemotaxis}

C. elegans can sense and respond to a variety of environmental stimuli through their sensory neurons. Specifically, the ASE amphid neurons are specialized to detect soluble chemicals, including cations $\left(\mathrm{Na}^{+}, \mathrm{K}^{+}\right)$, anions $\left(\mathrm{Cl}^{-}\right)$, basic $\mathrm{pH}$, cyclical nucleotides, certain amino acids, and other small molecules, including biotin and serotonin (Ward, 1973; Bargmann and Horvitz, 1991; Bargmann, 2006). Functional ASE cilia enable worms to migrate along $\mathrm{NaCl}$ gradients and accumulate in regions of their preferred salt concentration (Luo et al., 2014). Previous work found that mutant che-12(mn389) animals were unable to chemotax along a $\mathrm{NaCl}$ gradient and showed concomitant defects in dye uptake, an assay commonly used to examine the fidelity of cilia in C. elegans (Bacaj et al., 2008). We asked whether completely deleting che-12 or mutating the CHE-12 TOG domains would also compromise chemotaxis along a $\mathrm{NaCl}$ gradient, as well as the ability to take up dye.

We used an established chemotaxis assay in which worms were placed in the center of an agar plate containing either an isocratic $30 \mathrm{mM} \mathrm{NaCl}$ concentration (control plate) or a linear 0-60 mM NaCl concentration gradient (Luo et al., 2014; Figure 9A). As expected, WT worms were uniformly distributed on control plates but accumulated in the high-salt area of plates containing the linear $\mathrm{NaCl}$ gradient (Figure 9B). CHE-12::GFP and GFP::CHE-12 strains also accumulated in the high-salt area but were somewhat reduced in this bias compared with WT. Examination of the tagRFP::PH control strain used to analyze the effect of unc-119 replacement on phenotypes showed a similar reduction in chemotactic behavior, suggesting that the slightly reduced chemotaxis observed in the gfp-tagged che-12 strains is due to unc-119 replacement. In stark contrast to the WT and control strains, the distribution of $\Delta$ che-12 worms on gradient plates was not different from their distribution on isocratic control plates, demonstrating that these animals are unable to sense and respond to a $\mathrm{NaCl}$ gradient. The GFP::CHE-12 TOG-1'2' $3^{\prime} 4^{\prime}$ mutant strain phenocopied the $\Delta c h e-12$ strain (Figure 9B), indicating that GFP::CHE-12 TOG-1' $2^{\prime} 3^{\prime} 4^{\prime}$, although properly localized, is nonfunctional as measured in this assay. Similar results were obtained using a dye-uptake assay to evaluate ciliary function, in which the $\Delta$ che-12 and GFP.:CHE-12 TOG-1'2'3'4' mutant strains showed dramatically reduced dye-uptake frequencies compared with the wild-type or CHE-12::GFP and GFP::CHE-12 strains (Figure 9, C-E). Thus, the effect of mutating the CHE-12 TOG domains on cilia length and ultrastructure correlates with amphid cilia-based chemotactic function in the context of the whole animal.

\section{DISCUSSION}

TOG domain-containing protein families ch-TOG and CLASP regulate MT dynamics in the cytoplasm during both interphase and cell division (Brittle and Ohkura, 2005; Sousa et al., 2007; Slep, 2009; Al-Bassam et al., 2010; Al-Bassam and Chang, 2011; Currie et al., 2011; Widlund et al., 2011; Patel et al., 2012; Leano et al., 2013; Fox et al., 2014). ch-TOG uses its array of five TOG domains to promote MT polymerization during interphase. CLASP has a trimeric TOG domain array that promotes MT pause in interphase. During mitosis, both the ch-TOG and CLASP families regulate spindle dynamics, facilitating proper segregation of sister chromatids between daughter cells. Both ch-TOG and CLASP require tubulin-binding activity across their TOG domain arrays in order to regulate MT dynamics. Although the activities of ch-TOG, CLASP, and a host of other cytoplasmic regulators of MT dynamics have been extensively studied, little is known about factors that regulate ciliary MTs. We identified Crescerin as a novel TOG domain array-containing protein family through structural characterization of its second TOG domain. We find that Crescerin1 localizes to primary cilia in mammalian cells and uses its TOG domain array to promote MT polymerization in vitro. In C. elegans, we showed that the Crescerin family member CHE-12 requires canonical TOG tubulin-binding determinants for proper sensory cilia development. This highlights the emerging paradigm of MT regulators that mechanistically employ arrayed TOG domains with distinct structures and tubulin-binding activities to differentially regulate MT dynamics, whether during interphase or mitosis or in specialized organelles like the primary cilium.

Concordant with the ability of Crescerin 1 to promote MT polymerization in vitro, we find that Crescerin1 TOG2 is most similar to TOG1 from the ch-TOG family of MT polymerases. However, Crescerin1 TOG2 does have unique features, including the presence of a $\beta$-hairpin at its $\mathrm{C}$-terminal region that makes extensive contacts with HRs C-E and contributes to domain stability. The primary sequences of Crescerin's four TOG domains bear low sequence identity to one another and to TOG domains from ch-TOG and CLASP families. Comparing TOG domain structures, we find that the tubulin-binding surface of many of these domains adopts different conformations, which imply different tubulin-binding modes, as well as potential effects on the curvature of the tubulin heterodimer. Our data demonstrate that Crescerin1 TOG domain pairs promote tubulin polymerization in vitro. Studies analyzing the ability of ch-TOG family TOG domains to promote tubulin polymerization in vitro found that a pair of TOG domains (TOG1 and TOG2 from yeast Stu2 or Drosophila Msps) are required for this activity, whereas the individual TOG domains are not sufficient (Slep and Vale, 2007; Ayaz et al., 2012; Fox et al., 2014). In contrast, a single TOG domain (TOG2) from human CLASP1 was sufficient to promote tubulin polymerization in vitro, suggesting that it either stabilized a conformation of tubulin more amenable for polymerization or aided in lattice contacts through a direct or allosteric mechanism (Leano et al., 2013). Of interest, Crescerin TOG2 and TOG4 are also able to individually increase MT polymerization rates in vitro, similar to human CLASP1 TOG2 (Leano et al., 2013). The structural mechanism underlying the ability of these individual TOG domains to promote tubulin polymerization remains to be determined. In cells, we find that the central linker with the C-terminal TOG(3+4) pair, when overexpressed, can bind the lattice of cytoplasmic MTs. This parallels similar effects observed when a linker-TOG3-4 and a linkerTOG5 construct from the Drosophila ch-TOG family member Msps were overexpressed, yielding MT lattice association that was dependent on determinants in both the preceding linker and the TOG domain(s) (Currie et al., 2011). In contrast, Msps TOG1 and TOG2 do not appear to promote MT lattice association but have been shown to interact with free tubulin heterodimers over gel filtration, an activity that Msps TOG3 and TOG4 lack (Slep and Vale, 2007; Fox et al., 2014). This suggests that different TOG domains in the Msps array recognize different tubulin states: free tubulin versus polymerized tubulin. Collectively, this leads us to hypothesize that the Crescerin TOG array may also be polarized with differential tubulinbinding activity, in which the N-terminal TOG domains bind and promote tubulin incorporation into growing MTs, whereas the C-terminal TOG pair affords MT association. 

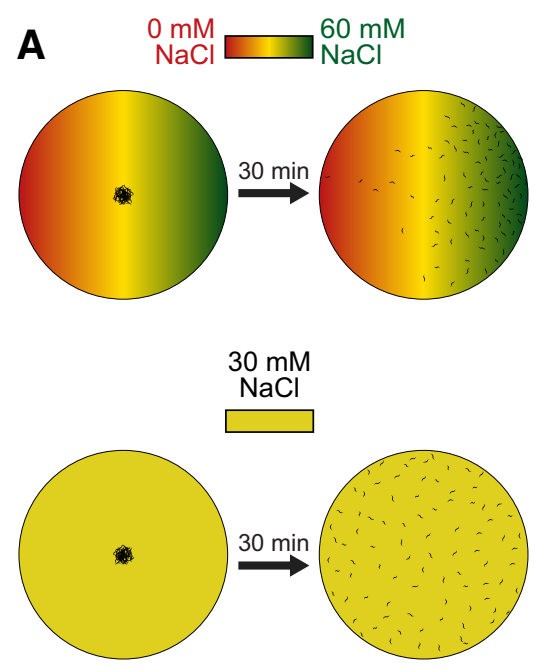

C

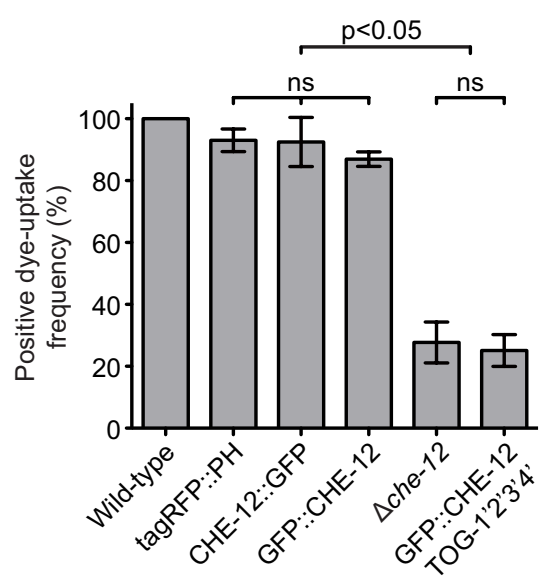

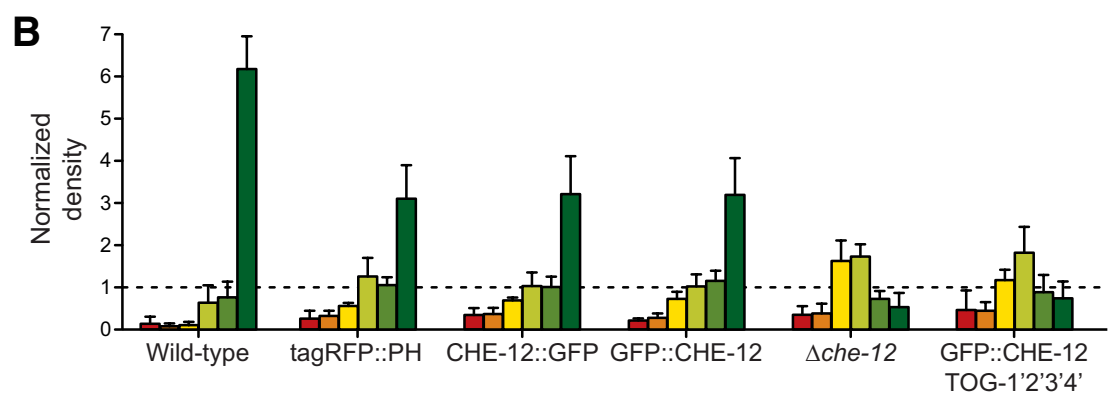

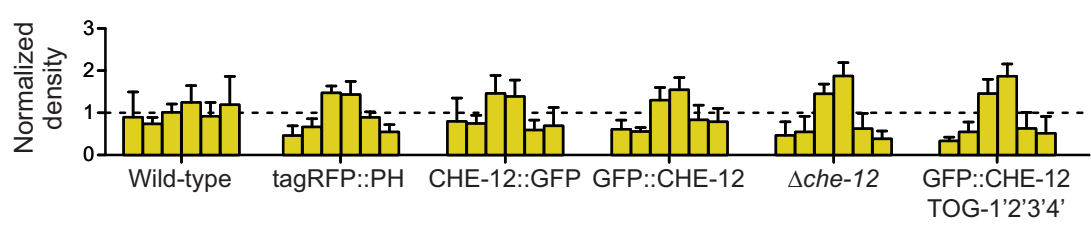

D

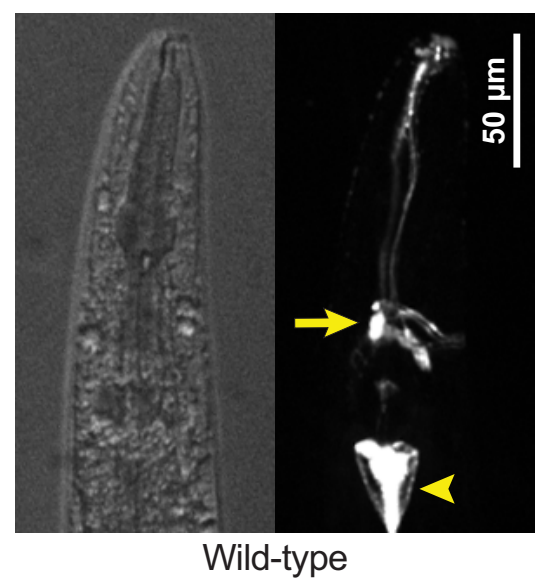

E

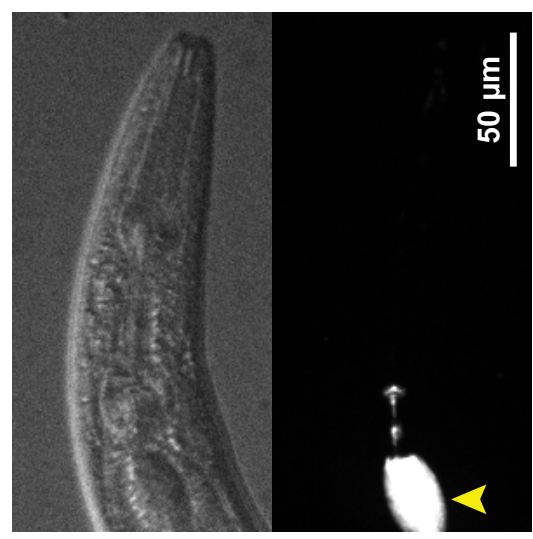

GFP::CHE-12 TOG-1'2'3'4'

FIGURE 9: Deleting che-12 or mutating tubulin-binding residues in CHE-12 TOG domains affects amphid cilia function.

(A) Schematic diagram of $C$. elegans chemotaxis assay on a $0-60 \mathrm{mM} \mathrm{NaCl}$ gradient (red-green) or $30 \mathrm{mM} \mathrm{NaCl}$ isocratic (yellow) agar plates. Worms are placed at the plate center and migration scored after $30 \mathrm{~min}$. WT animals preferentially migrate toward $60 \mathrm{mM} \mathrm{NaCl}$ on the gradient plate but distribute uniformly on an isocratic plate. (B) Bar graph showing the normalized density (see Materials and Methods) of worms in different sections of the plate. Error bars indicate SDs over the course of four or five experiments. (C) Quantitated results of Dil uptake assay. Error bars indicate SDs from three independent experiments. (D, E) Images showing strong dye-filling in WT (D) C. elegans amphid neurons (yellow arrow) and no dye filling in GFP::CHE-12 TOG-1' $2^{\prime} 3^{\prime} 4^{\prime}$ amphid neurons (E). Gut staining due to feeding: yellow arrowhead. Scale bars, $50 \mu \mathrm{m}$.

Why is Crescerin specific to cilia, and how does this compare and contrast with the regulation of MT dynamics in the cytoplasm? Extensive work has characterized MAPs that regulate cytoplasmic MTs, including investigations that probe the roles and mechanisms of the TOG array proteins ch-TOG and CLASP. Although MAPs affect MT dynamics, a key underlying factor is the concentration of tubulin and its ability to diffuse throughout the cytoplasm. This contrasts with components at the cilium tip, where the singlet microtubule plus ends reside. In this diffusion-limited space, a small fraction of the tubulin that is present diffuses in from the cytoplasm while the major fraction is transported to the cilia distal tip via IFT to reach the concentration required for MT polymerization, particularly during the nonequilibrium phase of ciliogenesis, when the flux of IFT-coupled tubulin is greatest (Craft et al., 2015). Previous studies identified cilia-specific tubulin isoforms in C. elegans (Hao et al., 2011), as well as in mammals (Péchart et al., 1999; Jensen-Smith et al., 2003). These tubulin isotypes are coupled to IFT and are actively transported to the distal tip (Hao et al., 2011; Bhogaraju et al., 2013). Little is known about the critical concentration of these tubulin isotypes or their dynamics, but models that predict how cilia of fixed length are generated and maintained rely on specific parameters that include the flux of tubulin to and from the cilia tip, as well as the parameters of ciliary microtubule dynamics (Ludington et al., 2015). Recent work found that the tubulin diffusion rate is slower at the cilia tip than at other ciliary regions, which suggests that nonpolymerized tubulin engages in macromolecular interactions with ciliary tip components (Craft et al., 2015). The differential nature of tubulin isotype and availability, as well as the difference in MT dynamics in the cytoplasm versus the distal cilia tip, suggests that additional, cilia-specific MT regulators are required to regulate ciliary MTs. We suggest 
that these differential requirements underlie the need for Crescerin in the cilium. Just as ch-TOG and CLASP are part of larger regulatory complexes, we anticipate that Crescerin interacts with other regulators of ciliary MT dynamics, coordinating phases of polymerization and depolymerization, driving polymerization during ciliogenesis, or oscillating between short phases of polymerization and depolymerization during the maintenance phase to retain a cilium of proper length.

With these observations, we reclassify Crescerin as a third conserved TOG domain-containing protein family found in ciliated and flagellated eukaryotes, specialized for MT regulation in cilia. In C. elegans, CHE-12 expression is restricted to a small set of sensory neurons that have a simple rod-shaped cilium. Further work is necessary to fully understand Crescerin 1 and Crescerin2 expression and function in mammalian systems, where different tissues have diversified cilia that perform highly specialized functions. Future experiments will examine whether Crescerin is associated with IFT particles, how it affects cilia MT dynamics, and whether it serves to deliver cilia-specific tubulin isotypes to the distal cilia tip.

\section{MATERIALS AND METHODS}

\section{Sequence analysis and phylogenetic tree}

Homologues of Crescerin from different organisms in the National Center for Biotechnology Information (NCBI) database were identified using the BLASTP algorithm (Altschul et al., 1990). The NCBI accession numbers of Crescerin homologues used for sequence alignments are given in Supplemental Table S1. Secondary structure predictions were performed using Jpred 3 (Cole et al., 2008) and PSIPRED servers (Jones, 1999; Buchan et al., 2013). Phylogenetic and molecular evolutionary analyses were conducted using integrated algorithms at the server phylogeny.lirmm.fr/phylo_cgi/index .cgi (Dereeper et al., 2008) and MEGA, version 6 (Tamura et al., 2013). Sequence alignment was carried out using the MUSCLE algorithm with the neighbor-joining method of clustering in MEGA. We used structural data from previously solved TOG domain structures to further optimize the alignment in the intra-HEAT loops. We constructed the initial tree using the maximum likelihood method implemented in the PhyML program (version 3.0). The LG substitution model was selected assuming an estimated proportion of invariant sites and four gamma-distributed rate categories to account for rate heterogeneity across sites. The gamma shape parameter was estimated directly from the data. Reliability for internal branch was assessed using the bootstrapping method (100 bootstrap replicates). We used this initial tree for tree inference using the SubtreePruning-Regrafting (extensive) algorithm with a very weak branch swap filter in MEGA, Version 6. Subsequent reliability for internal branch was assessed using the bootstrapping method (1000 bootstrap replicates). The final tree was prepared for publication using FigTree, version 1.4.0.

\section{Cloning and expression}

Mouse Crescerin1 TOG2 (residues 332-620) was cloned into pET28b (Novagen, EMD Millipore, Darmstadt, Germany), generating a thrombin-cleavable $\mathrm{N}$-terminal hexahistidine $\left(\mathrm{His}_{6}\right)$ tag. Crescerin1 TOG2 was transformed into BL21 DE3 Escherichia coli and grown in $6 \mathrm{I}$ of Luria broth $\left(50 \mu \mathrm{g} / \mathrm{ml}\right.$ kanamycin) at $37^{\circ} \mathrm{C}$ to an optical density of 0.6 at $600 \mathrm{~nm}$. The temperature was lowered to $15^{\circ} \mathrm{C}$ and protein expression induced with $100 \mu \mathrm{M}$ isopropyl- $\beta$-Dthiogalactoside (final concentration) for $16 \mathrm{~h}$. Cells were centrifuged at $2100 \times g$ for $10 \mathrm{~min}$ and pellets resuspended in $250 \mathrm{ml}$ buffer $\mathrm{A}(25 \mathrm{mM}$ Tris, $\mathrm{pH}$ 8.0, $300 \mathrm{mM} \mathrm{NaCl}, 10 \mathrm{mM}$ imidazole, $0.1 \% \beta$-mercaptoethanol $[\beta-\mathrm{ME}])$ and stored at $-20^{\circ} \mathrm{C}$. To produce selenomethionine-substituted Crescerin1 TOG2, expression was conducted in the B834 methionine auxotrophic E. colistrain and grown in L-selenomethionine-containing minimal media (MD12501 and MD12-502; Molecular Dimensions, Altamonte Springs, FL) according to the manufacturer's instructions.

\section{Protein purification}

Native and selenomethionine-substituted Crescerin1 TOG2 cell pellets were thawed and $0.5 \mathrm{mM}$ phenylmethylsulfonyl fluoride (PMSF) added, and the cells were lysed by sonication. After lysis, additional PMSF was added to $1 \mathrm{mM}$ final concentration, and the lysate was centrifuged at 23,000 $\times \mathrm{g}$ for $45 \mathrm{~min}$. Supernatant was loaded onto a $10-\mathrm{ml} \mathrm{Ni}{ }^{2+}$-nitriloacetic acid column (Qiagen, Venlo, Netherlands) and washed with $300 \mathrm{ml}$ of buffer $\mathrm{A}$, and TOG2 was eluted over a 250-ml linear gradient between buffers $A$ and $B$ (buffer B = buffer A supplemented with $290 \mathrm{mM}$ imidazole). TOG2-containing fractions were pooled, $\mathrm{CaCl}_{2}$ was added to $2.5 \mathrm{mM}$, and $0.1 \mathrm{mg}$ of bovine $\alpha$-thrombin (Haematologic Technologies, Essex Junction, VT) was added to remove the His 6 tag, leaving an N-terminal Gly-Ser-HisMet cloning artifact. Thrombin digest proceeded for at least $16 \mathrm{~h}$ at $4^{\circ} \mathrm{C}$. Digested protein was filtered over $0.5 \mathrm{ml}$ of benzamadine Sepharose (GE Healthcare, Buckinghamshire, United Kingdom) and exchanged into buffer C (25 mM 4-(2-hydroxyethyl)-1-piperazineethanesulfonic acid [HEPES], pH 7.0, 0.1\% $\beta$-ME) by dialysis. Protein was loaded onto a $10 \mathrm{ml}$ of SP-Sepharose fast flow column (GE Healthcare), washed with $200 \mathrm{ml}$ of buffer C, and eluted over a 250-ml linear gradient between buffers C and D (buffer D = buffer C supplemented with $1 \mathrm{M} \mathrm{NaCl}$ ). Protein factions were pooled, concentrated, and exchanged into $10 \mathrm{mM}$ HEPES, pH 7.5, $100 \mathrm{mM}$ $\mathrm{NaCl}$, and $0.1 \% \beta-\mathrm{ME}$ in a Millipore Ultrafree 10,000 MWCO concentrator to $15 \mathrm{mg} / \mathrm{ml}$, frozen in liquid nitrogen, and stored at $-80^{\circ} \mathrm{C}$. Purification of selenomethionine-substituted protein proceeded according to the aforementioned protocol.

\section{Crystallization}

Crescerin1 TOG2 was crystallized using the hanging-drop vapor diffusion method. A 2- $\mu$ l amount of protein (native and selenomethionine-substituted protein) at $10 \mathrm{mg} / \mathrm{ml}$ was added to an equal volume of a well solution containing $25 \%$ polyethylene glycol 3350 , $0.25 \mathrm{M}$ magnesium chloride, and 0.1 M HEPES, pH 7.5, and incubated at $20^{\circ} \mathrm{C}$. Crystals appeared overnight and reached maximum dimensions over the course of 2-3 d. Crystals were transferred to Fomblin oil (Sigma-Aldrich, St. Louis, MO) and flash frozen in liquid nitrogen.

\section{Data collection, structure determination, and refinement}

Isomorphous Crescerin1 TOG2 native and selenium peak singlewavelength anomalous dispersion data sets were collected on single crystals to resolutions of 2.1 and $2.3 \AA$, respectively. Diffraction data were collected at the Advanced Photon Source 22-ID beamline, Argonne National Laboratory, at $100 \mathrm{~K}$. Crescerin1 TOG2 crystals belong to the space group $\mathrm{P} 2{ }_{1} 2{ }_{1} 2$, with one molecule in the asymmetric unit. Data were indexed, integrated, and scaled using HKL2000 (Otwinowski and Minor, 1997). Selenium sites were identified and used to generate initial experimental, density-modified electron density maps (PHENIX; Grosse-Kunstleve and Adams, 2003; Terwilliger, 2004, 2007, 2008; Zwart et al., 2005; McCoy et al., 2007; Moriarty et al., 2009; Adams et al., 2010; Chen et al., 2010; Afonine et al., 2012). Initial models were built using AutoBuild (PHENIX), followed by reiterative manual building in Coot and refinement runs using phenix.refine (PHENIX). The selenomethioninesubstituted TOG2 structure was refined against a MLHL target 
function, and the model was converted to native methionine and refined against native data using a $\mathrm{ML}$ target function to $2.2-\AA \AA$ resolution. Refinement was monitored using a Free $\mathrm{R}$, based on $10 \%$ of the data randomly excluded from refinement. No clear electron density was evident for $\mathrm{N}$-terminal residues 332-344, HR A loops residues 361-365, HR F residues 558-562, and the C-terminal residues 595-620. Lysine K380 was modeled as alanine. Information regarding data statistics, model building, and refinement is given in Supplemental Table S3. Atomic coordinates have been deposited in the Protein Data Bank under accession code 5DN7.

\section{Circular dichroism}

The CD spectra of Crescerin 1 TOG domains were collected at 20 and $94^{\circ} \mathrm{C}$ using a Chirascan-plus CD spectrometer (Applied Photophysics, Leatherhead, United Kingdom). Each protein sample was diluted to a final concentration of $0.1 \mathrm{mg} / \mathrm{ml}$ in $10 \mathrm{mM}$ sodium phosphate buffer ( $\mathrm{pH} 7.5)$ and $50 \mathrm{mM} \mathrm{NaF}$. The spectra were recorded from 260 to $190 \mathrm{~nm}$ with a step size of $0.5 \mathrm{~nm}$ using a cuvette of $1 \mathrm{~mm}$ path length. The time per point was kept at $1.25 \mathrm{~s}$. A baseline control CD spectrum was taken and subtracted from each spectrum. All spectra were smoothed in the Chirascan-plus software with a moving window of five points. In $C D$ thermal melt experiments, data were collected at $1{ }^{\circ} \mathrm{C}$ intervals from 20 to $94^{\circ} \mathrm{C}$ at 208 - and $222-n m$ wavelengths.

\section{In vitro MT polymerization assay}

Crescerin1 constructs for MT polymerization assays (TOG1, 67-317; TOG2, 332-620; TOG2-Y364E; TOG3, 1243-1491; TOG4, 15331776; TOG4-F1559E; TOG(1+2), 67-620; and TOG(3+4), 12431776) were cloned into E. coli expression vectors, expressed, and purified in a manner similar to that for TOG2 as detailed earlier. Tubulin was purified from bovine brain following protocols established by the Mitchison lab (http://mitchison.med.harvard.edu/protocols/ microtubules/Large\%20Scale\%20Tubulin\%20Preparation.pdf). Tubulin polymerization was monitored using a SPEX Fluorolog-3 spectrophotometer (Horiba Jobin Yvon, Edison, NJ) in T-format, high-voltage mode. The excitation and detection wavelengths were set at $350 \mathrm{~nm}$ and the excitation and emission slits at 0.5 and 0.75 $\mathrm{nm}$, respectively. The cuvette holder was maintained at $37^{\circ} \mathrm{C}$. Clarified tubulin samples (18 $\mu \mathrm{M}$ final concentration) were prepared in the presence or absence of Crescerin1 TOG domain constructs each at $3 \mu \mathrm{M}$ final concentration in polymerization buffer $(50 \mathrm{mM} 2-(\mathrm{N}-$ morpholino)ethanesulfonic acid [MES], pH 6.6, 3.4 M glycerol, 5 mM dithiothreitol, $1 \mathrm{mM}$ ethylene glycol tetraacetic acid [EGTA], $5 \mathrm{mM}$ $\mathrm{MgSO}_{4}, 1 \mathrm{mM} \mathrm{GTP}$ ) and incubated on ice for $8 \mathrm{~min}$. The $300-\mu \mathrm{l}$ samples were injected into a quartz cuvette of $4 \mathrm{~mm}$ path length at room temperature and then immediately placed into the $37^{\circ} \mathrm{C}$ cuvette holder. Scattering was recorded at $350 \mathrm{~nm}$ in 1-s intervals over $1500 \mathrm{~s}$. Spikes in scattering within the first $100 \mathrm{~s}$ were the result of samples equilibrating to $37^{\circ} \mathrm{C}$.

\section{Mammalian expression plasmids}

For constitutive expression in mammalian cells driven by the cytomegalovirus (CMV) immediate early promoter, full-length and truncated mouse Crescerin 1 constructs (Figures 3, B and C, and 7) were cloned into pcDNA-DEST47 and pcDNA-DEST53 plasmids using the Gateway cloning system (Invitrogen, Thermo Fisher Scientific, Waltham, MA), generating C-terminal and N-terminal GFP fusion proteins, respectively.

\section{Cell culture and transfection}

IMCD3 cells were cultured in DMEM:F-12 (1:1) (Gibco, Thermo Fisher Scientific, Waltham, MA) supplemented with $10 \%$ fetal bovine serum (FBS; Gibco) and $1 \times$ Anti-Anti (Gibco) at $37^{\circ} \mathrm{C}$ and $5 \% \mathrm{CO}_{2}$. HEK-293 cells were cultured in DMEM (Gibco) supplemented with $10 \% \mathrm{FBS}$ and $1 \times$ Anti-Anti at $37^{\circ} \mathrm{C}$ and $5 \% \mathrm{CO}_{2}$. For transfection, IMCD3 and HEK-293 cells were seeded in six-well plates and transfected when they reached $70-80 \%$ confluence. At $1 \mathrm{~h}$ before transfection, the media in the cell culture plates were replaced with either Opti-MEM (IMCD3) or nonsupplemented DMEM (HEK-293). Cells were transfected with $2.5-3 \mu \mathrm{g}$ of the plasmid DNA per well of a six-well plate, using either polyethelenimine or Lipofectamine 3000 (Invitrogen) transfection reagents for HEK-293 and IMCD3 cells, respectively. After $6-8 \mathrm{~h}$ of transfection, the cells were seeded on glass coverslips in low-serum-supplemented $(0.25 \%$ FBS) DMEM. HEK-293 cells were serum starved for 24-48 $\mathrm{h}$ and IMCD3 cells were serum starved for $4 \mathrm{~d}$ to promote growth arrest and induce ciliogenesis. For MT lattice-binding assays, HEK-293 cells (not serum starved) were fixed $24 \mathrm{~h}$ after transfection.

\section{Immunofluorescence microscopy of mammalian cells}

For visualization of primary cilia in mammalian cells, the cells were first treated with PHEM buffer (60 mM 1,4-piperazinediethanesulfonic acid [PIPES], $21 \mathrm{mM}$ HEPES, $10 \mathrm{mM}$ EGTA, $2 \mathrm{mM} \mathrm{MgCl}$, pH 7.5) containing $4 \%$ formaldehyde for $20 \mathrm{~s}$, followed by permeabilization in PHEM buffer supplemented with $0.2 \%$ Triton X-100 for 2 min. The cells were then fixed in fresh PHEM buffer supplemented with $4 \%$ formaldehyde for $20 \mathrm{~min}$ and then blocked with $10 \mathrm{mg} / \mathrm{ml}$ BSA solution dissolved in PHEM buffer supplemented with 0.2\% Triton X-100. Polyclonal antibodies against mouse Crescerin 1 were raised in guinea pig (Pocono Rabbit Farm and Laboratory, Canadensis, PA) using purified fragments of TOG1 and TOG2 as antigen. Mouse monoclonal anti-acetylated tubulin antibody (clone 6-11B-1, T7451; SigmaAldrich) was used for labeling the primary cilium, and anti-pericentrin antibody raised in rabbit (ab4448; Abcam, Cambridge, United Kingdom) was used for labeling centrioles and basal bodies. Mouse monoclonal anti- $\alpha$-tubulin antibody (clone DM1A, T6199; SigmaAldrich) was used for labeling microtubules. Nuclei were stained with 4',6-diamidino-2-phenylindole (Molecular Probes, Invitrogen). All samples were mounted with mounting medium $(20 \mathrm{mM}$ Tris, $\mathrm{pH} 8.5,90 \%$ glycerol, $50 \mathrm{mg} / \mathrm{ml}$-propyl gallate). Images were collected on an inverted epifluorescence microscope (Eclipse Ti; Nikon, Tokyo, Japan) using a 100x objective. Images were analyzed using NIS-Elements imaging software and ImageJ (National Institutes of Health, Bethesda, MD).

\section{C. elegans culture and strain construction}

C. elegans strains were kept at $20^{\circ} \mathrm{C}$ and fed E. coli OP50 bacteria except where noted. Strain N2 was used as WT. To label the ASER neuron for imaging, we generated extrachromosomal arrays by injecting a plasmid encoding Pgcy-5::mCherry (a gift from Shai Shaham, Rockefeller University) together with the plasmid pRF4, which carries the dominant rol-6(su1006) marker (Mello et al., 1991). Supplemental Table S2 shows a list of all strains generated and used in this study. All new che-12 alleles reported in this study were generated using Cas9-triggered homologous recombination (Dickinson et al., 2013). For each modification, we built a homologous repair template comprising the desired edited nucleotide sequence flanked on each side by $1.5 \mathrm{~kb}$ of unmodified genomic sequence. Each repair template also contained an unc-119(+) selectable marker flanked by LoxP sites. In addition, we generated a Cas9sgRNA construct by inserting the desired target sequence into the vector pDD162 (Dickinson et al., 2013) using site-directed mutagenesis. Cas9 target sites were selected using the CRISPR design tool available at crispr.mit.edu/ and are listed in Supplemental Table S2. 
Young adults carrying an unc-119(ed3) mutant allele were injected with a plasmid mixture containing $50 \mathrm{ng} / \mu \mathrm{l}$ Cas9-sgRNA plasmid, $10 \mathrm{ng} / \mu \mathrm{l}$ homologous repair template, $10 \mathrm{ng} / \mu \mathrm{l}$ pMA122 (Phsp16.41::PEEL-1), $10 \mathrm{ng} / \mu \mathrm{l}$ pGH8 (Prab-3::mCherry), $5 \mathrm{ng} / \mu \mathrm{l}$ pCFJ104 (Pmyo-3::mCherry), and $2.5 \mathrm{ng} / \mu \mathrm{l}$ pCFJ90 (Pmyo-2::mCherry). Injected animals were placed at $25^{\circ} \mathrm{C}$ for $8-12 \mathrm{~d}$ and then heat shocked for $4 \mathrm{~h}$ at $34^{\circ} \mathrm{C}$ to induce expression of the PEEL-1 toxin, which kills animals carrying extrachromosomal arrays. Plates carrying non-uncoordinated animals that survived heat shock and lacked the mCherry coinjection were considered candidate knockout/ knock-in founders, and single worms from these plates were picked to establish lines. Genomic DNA was isolated from each line, and the presence of the desired genome modification was confirmed by PCR and sequencing. Only a single line from each independent plate was kept.

Supplemental Figure S3 shows the strategy for modifying the che-12 locus using homologous recombination. To generate che12-knockout alleles, we designed a homologous repair template to delete the entire che-12 coding sequence and replace it with gfp. This construct was injected into young adults of strain HT1593 for Cas9-triggered homologous recombination as described. To generate GFP-tagged WT and mutant che-12 alleles at the endogenous locus, we first removed the unc-119(+) selectable marker from the che-12-knockout strain LP177 by injecting Cre recombinase (Dickinson et al., 2013). The resulting uncoordinated animals were used for a second round of Cas9-triggered homologous recombination to reintroduce the WT or mutant che-12 gene.

\section{Fluorescence microscopy of $C$. elegans}

For fluorescence microscopy of $C$. elegans strains, adult worms were mounted on thin agar pads made of $2.5 \%$ agar dissolved in M9 medium. We used polystyrene nanoparticle beads (2.5\% by volume, 0.1- $\mu \mathrm{m}$ diameter; Polysciences, Warrington, PA) to immobilize the worms on the agar pad as described by Kim et al. (2013). The worms were imaged using a Nikon Eclipse Ti microscope equipped with 100x, 1.4 numerical aperture (NA) objective and Yokogawa (Tokyo, Japan) CSU-10 spinning disk head.

electron microscope operating at 80 kV (Carl Zeiss Microscopy, Peabody, MA), and digital images were acquired using a Gatan Orius SC1000 CCD Digital Camera with Digital Micrograph 3.11.0 (Gatan, Pleasanton, CA).

\section{Chemotaxis assays}

Chemotaxis plates with a linear $\mathrm{NaCl}$ gradient were prepared according to an established protocol (Luo et al., 2014). Briefly, $2 \%$ agar solutions in chemotaxis buffer $(5 \mathrm{mM}$ phosphate, $1 \mathrm{mM} \mathrm{CaCl}, 1 \mathrm{mM}$ $\mathrm{MgSO}_{4}$ ), with or without $60 \mathrm{mM} \mathrm{NaCl}$, were prepared and sterilized by autoclaving. Before pouring, each Petri dish (circular, $8.5-\mathrm{cm}$ diameter) was labeled to mark the high-salt and low-salt ends and six parallel sections of equal width perpendicular to the gradient axis. The plates were uniformly elevated at the low-salt end. Then $20 \mathrm{ml}$ of high-salt agar (containing $60 \mathrm{mM} \mathrm{NaCl}$ ) was poured into the plate and allowed to harden. This created a sloped agar block with maximum height at the high-salt end and barely covering the surface at the low-salt end. The plates were then laid flat, and $20 \mathrm{ml}$ of the lowsalt agar $(0 \mathrm{mM} \mathrm{NaCl})$ was poured over the high-salt agar block to create a horizontal surface. Plates were incubated for $16 \mathrm{~h}$ to allow $\mathrm{NaCl}$ to diffuse, creating a $0-60 \mathrm{mM} \mathrm{NaCl}$ gradient across the agar surface. Control isocratic chemotaxis plates were prepared using sterile chemotaxis agar supplemented with $30 \mathrm{mM} \mathrm{NaCl}$.

The $C$. elegans strains were grown for $3 \mathrm{~d}$ on nematode growth media plates seeded with bacteria to obtain a synchronous adult population. The worms were washed off the plates in chemotaxis buffer and collected in microcentrifuge tubes. The worms were allowed to settle to the bottom of the tube and washed three times with chemotaxis buffer to wash off the bacteria, embryos and juveniles. From 150 to 300 adult worms, resuspended in $20 \mu \mathrm{l}$ of chemotaxis buffer, were then pipetted into the plate center. Timing for the chemotaxis assay was initiated when the buffer evaporated completely and the worms started crawling on the agar surface. After $30 \mathrm{~min}$, the plates were cooled to $4^{\circ} \mathrm{C}$ to paralyze the worms, and the number of worms in each of six sectors along the plate's linear $\mathrm{NaCl}$ gradient were counted. We determined the normalized worm density for each sector, which we define as follows: Normalized density $=\frac{\text { Number of worms on sector } X / \text { Total number of worms on the plate }}{\text { Area of sector } X / \text { Total area of the plate }}$

\section{Transmission electron microscopy of $C$. elegans}

Adult C. elegans hermaphrodites were washed off agar plates with M9 buffer and collected into 1.5-ml microcentrifuge tubes. The worms were allowed to settle to the bottom of the tube and washed in M9 buffer to wash off the bacteria and then were fixed in $2 \%$ paraformaldehyde $/ 2.5 \%$ glutaraldehyde in $0.1 \mathrm{M}$ sodium cacodylate buffer, $\mathrm{pH} 7.4$, with $0.05 \% \mathrm{CaCl}_{2}$. Samples were microwave irradiated using a PELCO BioWave Pro microwave system (Ted Pella, Redding, CA) at $450 \mathrm{~W}$ to a temperature of $40^{\circ} \mathrm{C}$, allowed to cool to room temperature, and stored at $4^{\circ} \mathrm{C}$ for several days before processing. After several washes in $0.1 \mathrm{M}$ sodium cacodylate buffer, the samples were postfixed in $1 \%$ osmium tetroxide/1.25\% potassium ferrocyanide in $0.1 \mathrm{M}$ sodium cacodylate buffer for $1 \mathrm{~h}$, followed by rinses in deionized water. (LP177 worms were encapsulated in agarose before the next step.) Samples were dehydrated in a graded series of ethanol solutions $(30,50,75 \% 90,100,100)$, followed by two changes in propylene oxide, and infiltration and embedment in Spurr's low-viscosity epoxy resin (Polysciences). Ultrathin sections (70-80 nm) were cut using a diamond knife, mounted onto 200 mesh copper grids or Formvar carbon-coated copper slot grids, and stained with $4 \%$ aqueous uranyl acetate and Reynold's lead citrate. Samples were observed using a LEO EM910 transmission
By this measure, when the animals are uniformly distributed throughout the plate, the normalized density for each sector will be equal to 1 .

\section{Dye-uptake assay}

Adult C. elegans hermaphrodites were washed off agar plates with $\mathrm{M} 9$ buffer and collected into $1.5-\mathrm{ml}$ microcentrifuge tubes. The worms were allowed to settle to the bottom of the tube and washed three times in M9 buffer to wash off the bacteria. The worms were then resuspended in M9 buffer containing 1,1'-dioctadecyl-3,3,3', 3'-tetramethylindocarbocyanine perchlorate (Dil; Anaspec, Fremont, CA) at a final concentration of $10 \mu \mathrm{g} / \mathrm{ml}$ and incubated at $20^{\circ} \mathrm{C}$ for $3 \mathrm{~h}$. Postincubation, the worms were washed again in M9 buffer and transferred on a new agar plate to remove excess dye. After $5 \mathrm{~min}$, the worms were mounted on thin agar pads made of $2.5 \%$ agar dissolved in M9 medium supplemented with $10 \mathrm{mM} \mathrm{NaN}_{3}$ to immobilize the animals. The worms were imaged using a Nikon Eclipse Ti microscope equipped with a 100x/1.4 NA objective and a Yokogawa CSU-10 spinning disk head. A total of $\sim 100$ worms were imaged for each strain over the course of three experiments. 


\section{ACKNOWLEDGMENTS}

We thank members of the Slep, Rogers, and Goldstein laboratories for reagents and insightful discussions, S. Shaham for the Pgcy-5::mCherry plasmid, C. Higgins for C. elegans strain LP193, the Advanced Photon Source staff and A. Tripathy for technical assistance, and V. Madden for assistance with transmission electron microscopy. Funding was provided by the March of Dimes (FY14-247; K.C.S.), the National Institutes of Health (R01GM094415 to K.C.S. and R01GM083071 to B.G.), and the National Science Foundation (IOS0917726; B.G.). A.D. is a Howard Hughes Medical Institute International Student Research Fellow. D.J.D. is supported by National Institutes of Health T32 CA009156 and a Howard Hughes Medical Institute Fellowship of the Helen Hay Whitney Foundation.

\section{REFERENCES}

Adams PD, Afonine PV, Bunkóczi G, Chen VB, Davis IW, Echols N, Headd JJ, Hung LW, Kapral GJ, Grosse-Kunstleve RW, et al. (2010). PHENIX: A comprehensive Python-based system for macromolecular structure solution. Acta Crystallogr D Biol Crystallogr 66, 213-221.

Afonine PV, Grosse-Kunstleve RW, Echols N, Headd JJ, Moriarty NW, Mustyakimov M, Terwilliger TC, Urzhumtsev A, Zwart PH, Adams PD (2012). Towards automated crystallographic structure refinement with phenix.refine. Acta Crystallogr D Biol Crystallogr 68, 352-367.

Akhmanova A, Hoogenraad CC, Drabek K, Stepanova T, Dortland B, Verkerk T, Vermeulen W, Burgering BM, De Zeeuw Cl, Grosveld F, Galjart N (2001). CLASPs are CLIP-115 and -170 associating proteins involved in the regional regulation of microtubule dynamics in motile fibroblasts. Cell 104, 923-935.

Al-Bassam J, Chang F (2011). Regulation of microtubule dynamics by TOG-domain proteins XMAP215/Dis1 and CLASP. Trends Cell Biol 21 604-614.

Al-Bassam J, Kim H, Brouhard G, van Oijen A, Harrison SC, Chang F (2010). CLASP promotes microtubule rescue by recruiting tubulin dimers to the microtubule. Dev Cell 19, 245-258.

Al-Bassam J, Larsen NA, Hyman AA, Harrison SC (2007). Crystal structure of a TOG domain: conserved features of XMAP215/Dis1-family TOG domains and implications for tubulin binding. Structure 15, 355-362.

Altschul SF, Gish W, Miller W, Myers EW, Lipman DJ (1990). Basic local alignment search tool. J Mol Biol 215, 403-410.

Ayaz P, Ye X, Huddleston P, Brautigam CA, Rice LM (2012). A TOG: $\alpha \beta$ tubulin complex structure reveals conformation-based mechanisms for a microtubule polymerase. Science 337, 857-860.

Bacaj T, Lu Y, Shaham S (2008). The conserved proteins CHE-12 and DYF11 are required for sensory cilium function in Caenorhabditis elegans. Genetics 178, 989-1002.

Bargmann Cl (2006). Chemosensation in C. elegans. WormBook 2009(Oct25), 1-29.

Bargmann $\mathrm{Cl}$, Horvitz HR (1991). Chemosensory neurons with overlapping functions direct chemotaxis to multiple chemicals in C. elegans. Neuron 7, 729-742.

Bhogaraju S, Cajanek L, Fort C, Blisnick T, Weber K, Taschner M, Mizuno N, Lamla S, Bastin P, Nigg EA, Lorentzen E (2013). Molecular basis of tubulin transport within the cilium by IFT74 and IFT81. Science 341, 1009-1012.

Brittle AL, Ohkura H (2005). Mini spindles, the XMAP215 homologue, suppresses pausing of interphase microtubules in Drosophila. EMBO J 24, 1387-1396.

Buchan DWA, Minneci F, Nugent TCO, Bryson K, Jones DT (2013). Scalable web services for the PSIPRED Protein Analysis Workbench. Nucleic Acids Res 41, W349-W357.

Chen VB, Arendall WB, Headd JJ, Keedy DA, Immormino RM, Kapral GJ, Murray LW, Richardson JS, Richardson DC (2010). MolProbity: all-atom structure validation for macromolecular crystallography. Acta Crystallogr D Biol Crystallogr 66, 12-21.

Christensen ST, Pedersen SF, Satir P, Veland IR, Schneider L (2008). The primary cilium coordinates signaling pathways in cell cycle control and migration during development and tissue repair. Curr Top Dev Biol 85, 261-301.
Cole C, Barber JD, Barton GJ (2008). The Jpred 3 secondary structure prediction server. Nucleic Acids Res 36, W197-W201.

Craft JM, Harris JA, Hyman S, Kner P, Lechtreck KF (2015). Tubulin transport by IFT is upregulated during ciliary growth by a cilium-autonomous mechanism. J Cell Biol 208, 223-237.

Currie JD, Stewman S, Schimizzi G, Slep KC, Ma A, Rogers SL (2011). The microtubule lattice and plus-end association of Drosophila Mini spindles is spatially regulated to fine-tune microtubule dynamics. Mol Biol Cell $22,4343-4361$.

De La Mora-Rey T, Guenther BD, Finzel BC (2013). The structure of the TOG-like domain of Drosophila melanogaster Mast/Orbit. Acta Crystallogr F Struct Biol Cryst Commun 69, 723-729.

Dereeper A, Guignon V, Blanc G, Audic S, Buffet S, Chevenet F, Dufayard JF, Guindon S, Lefort V, Lescot M, et al. (2008). Phylogeny.fr: robust phylogenetic analysis for the non-specialist. Nucleic Acids Res 36, W465-W469.

Dickinson DJ, Ward JD, Reiner DJ, Goldstein B (2013). Engineering the Caenorhabditis elegans genome using Cas9-triggered homologous recombination. Nat Methods 10, 1028-1034.

Drabek K, van Ham M, Stepanova T, Draegestein K, van Horssen R, Sayas CL, Akhmanova A, Ten Hagen T, Smits R, Fodde R, et al. (2006). Role of CLASP2 in microtubule stabilization and the regulation of persistent motility. Curr Biol 16, 2259-2264.

Fox JN, Currie JD, Rogers SL, Slep KC (2014). The XMAP215 family drives microtubule polymerization using a structurally diverse TOG array. Mol Biol Cell 25, 2375-2392.

Grosse-Kunstleve RW, Adams PD (2003). Substructure search procedures for macromolecular structures. Acta Crystallogr D Biol Crystallogr 59, 1966-1973.

Hao L, Thein M, Brust-Mascher I, Civelekoglu-Scholey G, Lu Y, Acar S, Prevo B, Shaham S, Scholey JM (2011). Intraflagellar transport delivers tubulin isotypes to sensory cilium middle and distal segments. Nat Cell Biol 13, 790-798.

Hoey DA, Downs ME, Jacobs CR (2012). The mechanics of the primary cilium: an intricate structure with complex function. J Biomech 45, 17-26.

Holm L, Rosenström P (2010). DALI server: conservation mapping in 3D. Nucleic Acids Res 38, W545-W549.

Ishikawa T (2012). Structural biology of cytoplasmic and axonemal dyneins. J Struct Biol 179, 229-234.

Jensen-Smith HC, Ludueña RF, Hallworth R (2003). Requirement for the betal and betalV tubulin isotypes in mammalian cilia. Cell Motil. Cytoskeleton 55, 213-220.

Jones DT (1999). Protein secondary structure prediction based on positionspecific scoring matrices. J Mol Biol 292, 195-202.

Kim E, Sun L, Gabel CV, Fang-Yen C (2013). Long-term imaging of Caenorhabditis elegans using nanoparticle-mediated immobilization. PLoS One 8, 1-6.

Kobayashi D, Takeda H (2012). Ciliary motility: the components and cytoplasmic preassembly mechanisms of the axonemal dyneins. Differentiation 83, S23-S29.

Leano JB, Rogers SL, Slep KC (2013). A cryptic TOG domain with a distinct architecture underlies CLASP-dependent bipolar spindle formation. Structure 21, 939-950.

Ludington WB, Ishikawa H, Serebrenik YV, Ritter A, Hernandez-Lopez RA, Gunzenhauser J, Kannegaard E, Marshall WF (2015). A systematic comparison of mathematical models for inherent measurement of ciliary length: how a cell can measure length and volume. Biophys J 108, 1361-1379.

Luo L, Wen Q, Ren J, Hendricks M, Gershow M, Qin Y, Greenwood J, Soucy ER, Klein M, Smith-Parker HK, et al. (2014). Dynamic encoding of perception, memory, and movement in a $C$. elegans chemotaxis circuit. Neuron 82, 1115-1128.

Marshall WF (2008). The cell biological basis of ciliary disease. J Cell Biol 180, 17-21.

Marshall WF, Nonaka S (2006). Cilia: tuning in to the cell's antenna. Curr Biol 16, R604-R614

Marshall WF, Rosenbaum JL (2001). Intraflagellar transport balances continuous turnover of outer doublet microtubules: Implications for flagellar length control. J Cell Biol 155, 405-414.

McCoy AJ, Grosse-Kunstleve RW, Adams PD, Winn MD, Storoni LC, Read RJ (2007). Phaser crystallographic software. J Appl Crystallogr 40, 658-674.

Mello CC, Kramer JM, Stinchcomb D, Ambros V (1991). Efficient gene transfer in C.elegans: extrachromosomal maintenance and integration of transforming sequences. EMBO J 10, 3959-3970. 
Moriarty NW, Grosse-Kunstleve RW, Adams PD (2009). Electronic ligand builder and optimization workbench (eLBOW): A tool for ligand coordinate and restraint generation. Acta Crystallogr D Biol Crystallogr 65 1074-1080.

Mukhopadhyay S, Lu Y, Shaham S, Sengupta P (2008). Sensory signalingdependent remodeling of olfactory cilia architecture in C. elegans. Dev Cell 14, 762-774.

Otwinowski Z, Minor W (1997). Processing of X-ray diffraction data collected in oscillation mode. Methods Enzymol 276, 307-326.

Patel K, Nogales E, Heald R (2012). Multiple domains of human CLASP contribute to microtubule dynamics and organization in vitro and in Xenopus egg extracts. Cytoskeleton 69, 155-165.

Péchart I, Kann ML, Levilliers N, Bré MH, Fouquet JP (1999). Composition and organization of tubulin isoforms reveals a variety of axonemal models. Biol Cell 91, 685-697.

Perkins LA, Hedgecock EM, Thomson JN, Culotti JG (1986). Mutant sensory cilia in the nematode Caenorhabditis elegans. Dev Biol 117, 456-487.

Rich DR, Clark AL (2012). Chondrocyte primary cilia shorten in response to osmotic challenge and are sites for endocytosis. Osteoarthritis. Cartilage 20, 923-930.

Satir P, Christensen ST (2007). Overview of structure and function of mammalian cilia. Annu Rev Physiol 69, 377-400.

Satir P, Christensen ST (2008). Structure and function of mammalian cilia. Histochem Cell Biol 129, 687-693.

Scholey JM (2003). Intraflagellar transport. Annu Rev Cell Dev Biol 19, 423-443.

Scholey JM (2008). Intraflagellar transport motors in cilia: moving along the cell's antenna. J Cell Biol 180, 23-29.

Slep KC (2009). The role of TOG domains in microtubule plus end dynamics. Biochem Soc Trans 37, 1002-1006.

Slep KC, Vale RD (2007). Structural basis of microtubule plus end tracking by XMAP215, CLIP-170, and EB1. Mol Cell 27, 976-991.
Sousa A, Reis R, Sampaio P, Sunkel CE (2007). The Drosophila CLASP homologue, Mast/Orbit regulates the dynamic behaviour of interphase microtubules by promoting the pause state. Cell Motil Cytoskeleton 64, 605-620.

Tamura K, Stecher G, Peterson D, Filipski A, Kumar S (2013). MEGA6: Molecular Evolutionary Genetics Analysis version 6.0. Mol Biol Evol 30, $2725-2729$

Terwilliger T (2004). SOLVE and RESOLVE: automated structure solution, density modification, and model building. J Synchrotron Radiat 11, 49-52.

Terwilliger TC, Grosse-Kunstleve RW, Afonine PV, Moriarty NW, Adams PD Read RJ, Zwart PH, Hung LW (2008). Iterative-build OMIT maps: map improvement by iterative model building and refinement without model bias. Acta Crystallogr D Biol Crystallogr 64, 515-524.

Terwilliger TC, Grosse-Kunstleve RW, Afonine PV, Moriarty NW, Zwart PH, Hung LW, Read RJ, Adams PD (2007). Iterative model building, structure refinement and density modification with the PHENIX AutoBuild wizard. Acta Crystallogr D Biol Crystallogr 64, 61-69.

Tilney LG, Gibbins JR (1968). Differential effects of antimitotic agents on the stability and behavior of cytoplasmic and ciliary microtubules. Protoplasma 65, 167-179.

Veland IR, Awan A, Pedersen LB, Yoder BK, Christensen ST (2009). Primary cilia and signaling pathways in mammalian development, health and disease. Nephron Physiol 111, p39-53.

Ward S (1973). Chemotaxis by the nematode Caenorhabditis elegans: identification of attractants and analysis of the response by use of mutants. Proc Natl Acad Sci USA 70, 817-821.

Widlund PO, Stear JH, Pozniakovsky A, Zanic M, Reber S, Brouhard GJ, Hyman AA, Howard J (2011). XMAP215 polymerase activity is built by combining multiple tubulin-binding TOG domains and a basic latticebinding region. Proc Natl Acad Sci USA 108, 2741-2746.

Zwart PH, Grosse-Kunstleve RW, Adams PD (2005). Xtriage and Fest: automatic assessment of $\mathrm{X}$-ray data and substructure structure factor estimation. CCP4 Newsl 43, 27-35. 